\title{
Triazabicyclodecene: an Effective Isotope Exchange Catalyst in $\mathrm{CDCl}_{3}$
}

\author{
Supporting Information
}

Cyrille Sabot ${ }^{\dagger}$ Kanduluru Ananda Kumar, ${ }^{\dagger}$ Cyril Antheaume, ${ }^{\ddagger}$ Charles Mioskowski ${ }^{\dagger}{ }^{\dagger}$

Laboratoire de Synthèse Bio-Organique, UMR 7175/LC1-CNRS, and Service Commun de RMN, IFR85, Université Louis Pasteur de Strasbourg, 74 route du Rhin, B.P 60024, 67401 Illkirch cedex, France

mioskow@aspirine.u-strasbg.fr

Table of contents

Experimental procedures $\quad$............................. 4

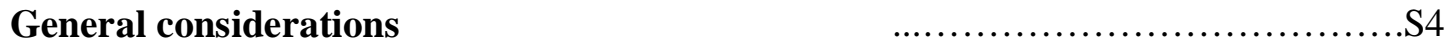

Representative procedure for deuteration reaction

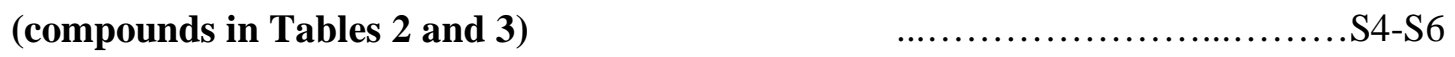

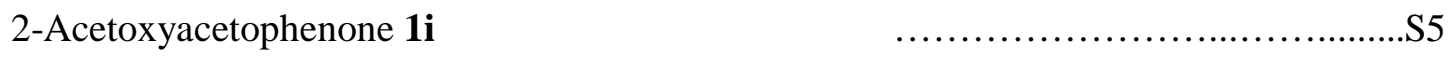

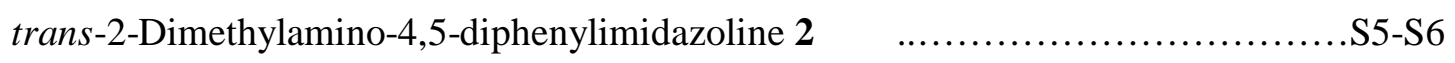

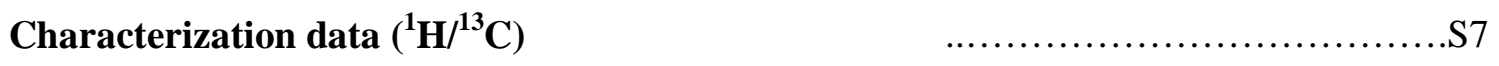

2-Acetoxyacetophenone $\mathbf{1 i} \quad$.............................. 
S-Methyltetramethylthiouronium iodide 2'

trans-2-Dimethylamino-4,5-diphenylimidazoline 2

Spectral data $\left({ }^{1} \mathbf{H} /{ }^{2} \mathbf{H}\right)$

TMG

4'-Methoxyacetophenone- $d_{3} \mathbf{1 a -} \boldsymbol{d}_{3}$

Acetophenone- $d_{3} \mathbf{1 b}-\boldsymbol{d}_{\mathbf{3}}$

S12-S15

4'-Nitroacetophenone- $d_{3}$ 1c- $\boldsymbol{d}_{3}$ S16

2-Methoxyacetophenone- $d_{2} \mathbf{1 d}-\boldsymbol{d}_{\mathbf{2}}$ S17

2-Methylacetophenone- $d_{2} \mathbf{1 e}-\boldsymbol{d}_{\mathbf{2}}$

2-Chloroacetophenone- $d_{2} \mathbf{1 f}-\boldsymbol{d}_{\mathbf{2}}$

2,2-Dimethylacetophenone- $d_{1} \mathbf{1 g}-\boldsymbol{d}_{\mathbf{1}}$

1-Phenylpropane-1,2-dione- $d_{3}: \mathbf{1 h}-\boldsymbol{d}_{\mathbf{3}}$

2-Acetoxyacetophenone- $d_{2} \mathbf{1 i}-\boldsymbol{d}_{\mathbf{2}}$ S22

Naphthylmethylketone- $\boldsymbol{d}_{\mathbf{3}} \mathbf{1} \mathbf{j}-\boldsymbol{d}_{\mathbf{3}}$ .$S 23$

2-Acetylpyridine- $d_{3} \mathbf{1 k}-d_{3}$ .$S 24$

2-Acetylthiophene- $d_{3} \mathbf{1 1}-\boldsymbol{d}_{\mathbf{3}}$ .$S 25$

Benzylacetone- $d_{5} \mathbf{1 m}-\boldsymbol{d}_{\mathbf{5}}$

S26-S28

Cyclodecanone- $d_{3}$ 1n- $\boldsymbol{d}_{4}$

S29-S31

1-Phenylacetylene- $d_{1}$ 3a- $d_{1}$

4-Phenylbut-1-yne- $\mathrm{d}_{1} \mathbf{3 b}-\boldsymbol{d}_{\mathbf{1}}$

1-Phenyl-2-methylacetylene- $\mathrm{d}_{3} 3 \mathbf{c}$ S34

2-Ethynyl-1-methylimidazole $\mathbf{3 d}-\boldsymbol{d}_{\mathbf{1}}$

S35-S36

Methyl-1-phenylacetate $4-\boldsymbol{d}_{\mathbf{2}}$ S37

3-Nitrofluorene $\mathbf{5}-\boldsymbol{d}_{\mathbf{2}}$ S38

Indene 6-d $\boldsymbol{d}_{\mathbf{3}}$ 
${ }^{1}$ H NMR study

References .............................S41 


\section{Experimental procedures}

\section{General considerations}

All substrates were commercially available and used without further purification except 2acetoxyacetophenone 1i synthesized from commercially available 2-hydroxyacetophenone. The trans-2-dimethylamino-4,5-diphenylimidazoline 2 was obtained in two steps from commercially available tetramethylthiourea. Deuterated chloroform used for deuterium incorporation reactions, contained $\mathrm{D}, 99.80 \%$ and $\mathrm{H}_{2} \mathrm{O}<0.01 \%$. ${ }^{1} \mathrm{H}$ and ${ }^{1} \mathrm{H}-\left\{{ }^{2} \mathrm{H}\right\}$ NMR spectral data were recorded at 600 or $300 \mathrm{MHz}$, on spectrometers using a software pulse program library. $\mathrm{CDCl}_{3}$ was used as lock and $\mathrm{CHCl}_{3}$ as internal standard $(7.27 \mathrm{ppm}) .{ }^{2} \mathrm{H}$ NMR spectral data were recorded at $46 \mathrm{MHz}$ and chemical shifts are reported relative to $\mathrm{CDCl}_{3}(7.27 \mathrm{ppm})$. HRMS analysis was performed in positive electrospray mode.

\section{Representative procedure for deuteration reaction (compounds in Tables 2 and 3):}

For compound 1b:

To a solution of $10 \mathrm{~mol} \%$ of TBD $(10 \mathrm{mg}, 0.072 \mathrm{mmol})$ in $3 \mathrm{~mL}$ of $\mathrm{CDCl}_{3}$, was added $\mathbf{1 b}(84$ $\mu \mathrm{L}, 0.72 \mathrm{mmol}$ ). The reaction mixture was stirred at room temperature for 12 hours and quenched with $1 \mathrm{~N} \mathrm{HCl}(1 \mathrm{~mL})$. The organic layer was washed with water $(2 \times 2 \mathrm{~mL})$, brine (1 $\mathrm{mL}$ ), dried over anhydrous $\mathrm{Na}_{2} \mathrm{SO}_{4}$ and filtered. Filtrate was concentrated to afford $\mathbf{1 b}-\boldsymbol{d}_{\mathbf{3}}$. The total incorporation yield was determined by ${ }^{1} \mathrm{H}$ NMR spectroscopy relative to the intensity of a non exchangeable proton in the molecule, or by GC-MS. For example, the global deuterium incorporation of compound $\mathbf{1 b}-\boldsymbol{d}_{\mathbf{3}}$ determined by GC-MS, was calculated as follow: $1 / 3 \times 5.3 \%+2 / 3 \times 28.5 \%+3 / 3 \times 65.6 \%=86.4 \%$ of total incorporation. Moreover, 
the site of deuterium incorporation in the substrate was determined by ${ }^{2} \mathrm{H}$ NMR spectroscopy relative to $\mathrm{CHCl}_{3}(7.27 \mathrm{ppm})$.

2-Acetoxyacetophenone $\mathbf{1 i}$

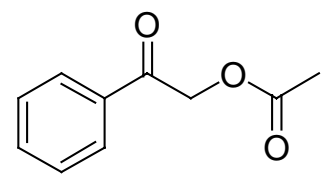

$1 \mathbf{i}$

To a solution of 2-hydroxyacetophenone $(1 \mathrm{~g}, 9.3 \mathrm{mmol})$ dissolved in pyridine $(40 \mathrm{~mL})$, dried over $3 \AA$ molecular sieves beforehand, was added acetyl chloride $(667 \mu \mathrm{L}, 9.3 \mathrm{mmol})$ by dropwise at $0{ }^{\circ} \mathrm{C}$. The reaction mixture was stirred at room temperature for 8 hours. The solution was filtered, and filtrate concentrated. The residue was dissolved with $1 \mathrm{~N} \mathrm{HCl}$ and the organic layer was extracted twice with diethylether. The combined organic layers were washed with brine and dried over anhydrous sodium sulfate, filtered and concentrated to afford a brown residue. The crude product was purified by chromatography on a silica gel column eluting with $20 \%$ ethyl acetate in cyclohexane to give $1.320 \mathrm{~g}$ ( $80 \%$ yield) of $\mathbf{1 i}$ as a yellow oil. Analytical data were in accordance with the previous literature report. ${ }^{1} R_{\mathrm{f}}=0.44$ (UV, Vanilline) in 30\% EtOAc/ Cyclohexane. ${ }^{1} \mathrm{H} \mathrm{NMR}\left(\mathrm{CDCl}_{3}, 300 \mathrm{MHz}\right): \delta 2.24(\mathrm{~s}, 3 \mathrm{H})$, $5.35(\mathrm{~s}, 2 \mathrm{H}), 7.45-7.55$ (t, J=7.5 Hz, 2H, Har), 7.60-7.70 (t, J=7.5 Hz, 1H, Har), 7.90-8.00 (t, $\mathrm{J}=7.5 \mathrm{~Hz}, 2 \mathrm{H}, \mathrm{Har}) .{ }^{13} \mathrm{C} \mathrm{NMR}\left(\mathrm{CDCl}_{3}, 75 \mathrm{MHz}\right): \delta 20.8,66.3,128.0,129.1,134.1,134.4$, 170.6, 192.4. IR (KRS-5): 2936, 1751, 1705, 1598, 1450, 1421, 1375, 1220. CIMS 179 $\left[\mathrm{M}+\mathrm{H}^{+}\right], 196\left[\mathrm{M}+\mathrm{NH}_{4}^{+}\right]$.

trans-2-Dimethylamino-4,5-diphenylimidazoline 2 


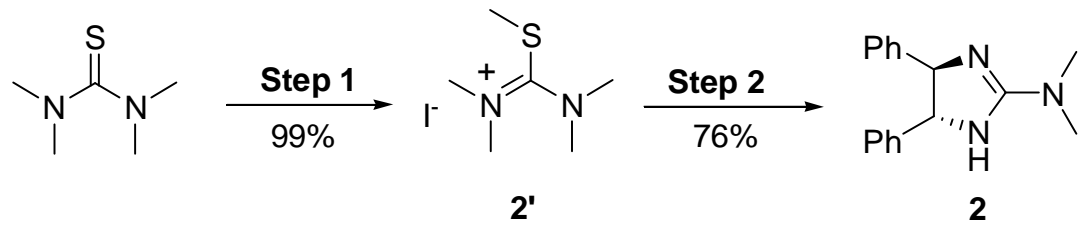

Step 1: S-Methyltetramethylthiouronium iodide 2'

To a solution of tetramethylthiourea $(1 \mathrm{~g}, 7.57 \mathrm{mmol})$ in $8 \mathrm{~mL}$ of dried methanol at $0{ }^{\circ} \mathrm{C}$ under argon, was added methyl iodide $(870 \mu \mathrm{L}, 14.09 \mathrm{mmol})$ by dropwise. After $16 \mathrm{~h}$ stirring at room temperature, the solvent was removed under reduced pressure and 2,065 $\mathrm{g}(99 \%$ yield) of 2' as a yellow solid was obtained (hygroscopic). ${ }^{2} \mathrm{RMN}{ }^{1} \mathrm{H}\left(300 \mathrm{MHz} ; \mathrm{CDCl}_{3}\right) \delta$ (ppm): 2.62 (s, 3H), 3.35 (s, 12H). $\mathrm{RMN}^{13} \mathrm{C}\left(75 \mathrm{MHz} ; \mathrm{CDCl}_{3}\right) \delta(\mathrm{ppm}): 18.6$, 45.3, 176.9.

Step 2: trans-2-Dimethylamino-4,5-diphenylimidazoline 2

To a solution of trans-1,2-diphenylethylenediamine $(1 \mathrm{~g}, 4.71 \mathrm{mmol})$ in $14 \mathrm{~mL}$ of dried acetonitrile, at $0{ }^{\circ} \mathrm{C}$, under argon, was added S-methyltetramethylthiouronium iodide 2' $(1.808 \mathrm{~g}, 6.59 \mathrm{mmol})$. The solution was stirred at room temperature for $2 \mathrm{~h}$ and further $2 \mathrm{~h}$ at reflux. After cooling, the solution was diluted with $50 \mathrm{~mL}$ of dichloromethane and treated by a solution $5 \%$ of sodium carbonate $(2 \times 50 \mathrm{~mL})$. The organic layer was dried over potassium carbonate, filtered and filtrate concentrated. After recrystallization (dichloromethane and a minimum of $n$-hexane), $953 \mathrm{mg}$ (76\% yield) of a white solid 2 was obtained. mp 202-203 ${ }^{\circ} \mathrm{C}$ $\mathrm{RMN}{ }^{1} \mathrm{H}\left(300 \mathrm{MHz} ; \mathrm{CDCl}_{3}\right) \delta(\mathrm{ppm}): 3.02(\mathrm{~s}, 6 \mathrm{H}), 4.69(\mathrm{~s}, 2 \mathrm{H}), 4.78(\mathrm{bs}, 1 \mathrm{H}), 7.15-7.48(\mathrm{~m}$, 10H). $\mathrm{RMN}{ }^{13} \mathrm{C}\left(50 \mathrm{MHz} ; \mathrm{CDCl}_{3}\right) \delta$ (ppm): 38.8, 74.0, 126.9, 127.7, 128.8, 143.8, 162.3. IR $(\mathrm{KRS}-5) \vee\left(\mathrm{cm}^{-1}\right):$ 3028, 2866, 2827, 1667, 1609, 1485, 1451. MS (Electrospray) 266.1612 $\mathrm{m} / \mathrm{z}(266.1657$ calcd for $\mathrm{M}+\mathrm{H})$. 


\section{Characterization data $\left({ }^{1} \mathrm{H} /{ }^{13} \mathrm{C}\right)$}

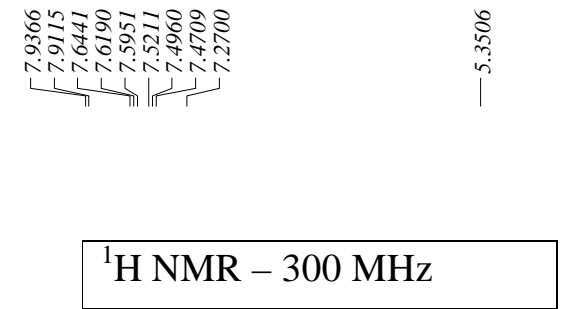

${ }^{1} \mathrm{H}$ NMR $-300 \mathrm{MHz}$

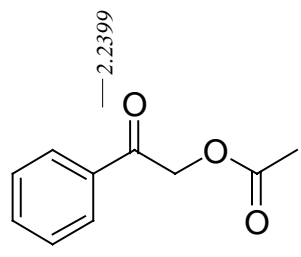

$1 \mathbf{i}$
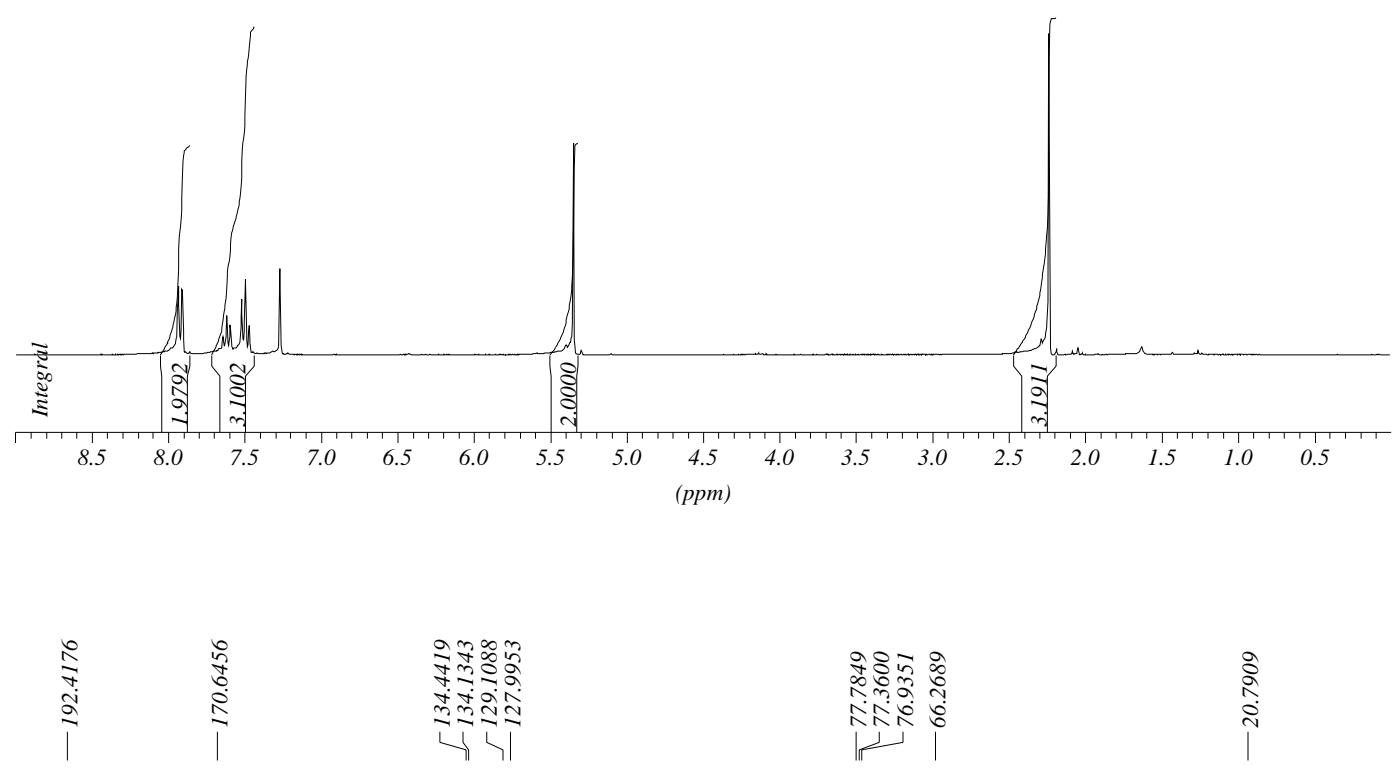

\section{${ }^{13} \mathrm{C}$ NMR $-75 \mathrm{MHz}$}

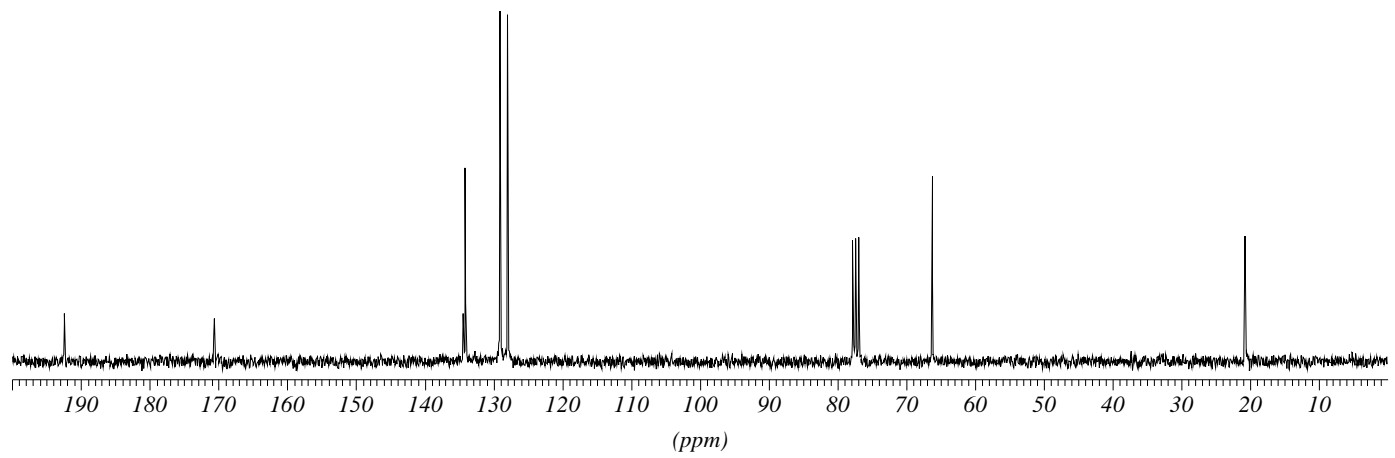



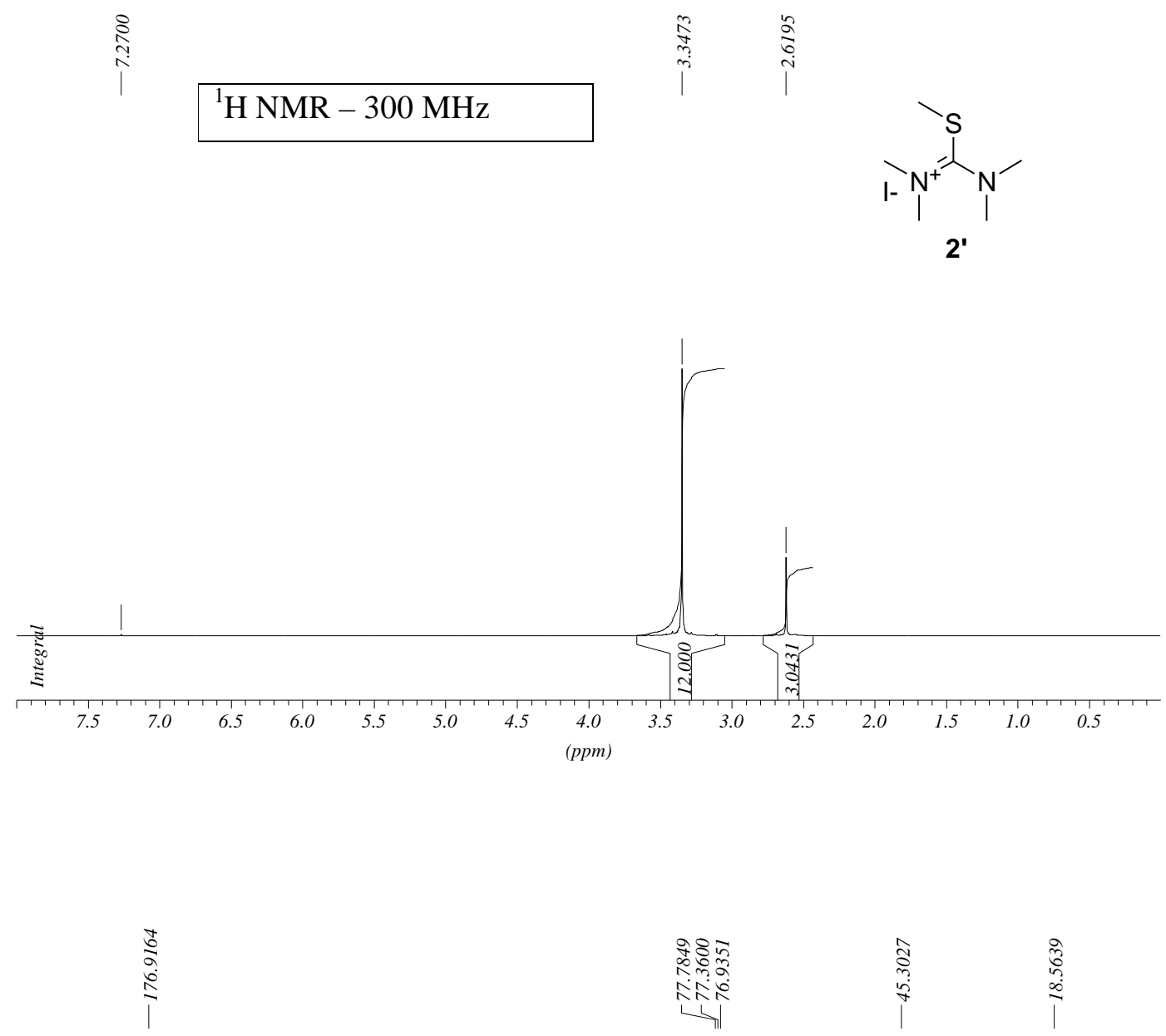

${ }^{13} \mathrm{C}$ NMR $-75 \mathrm{MHz}$

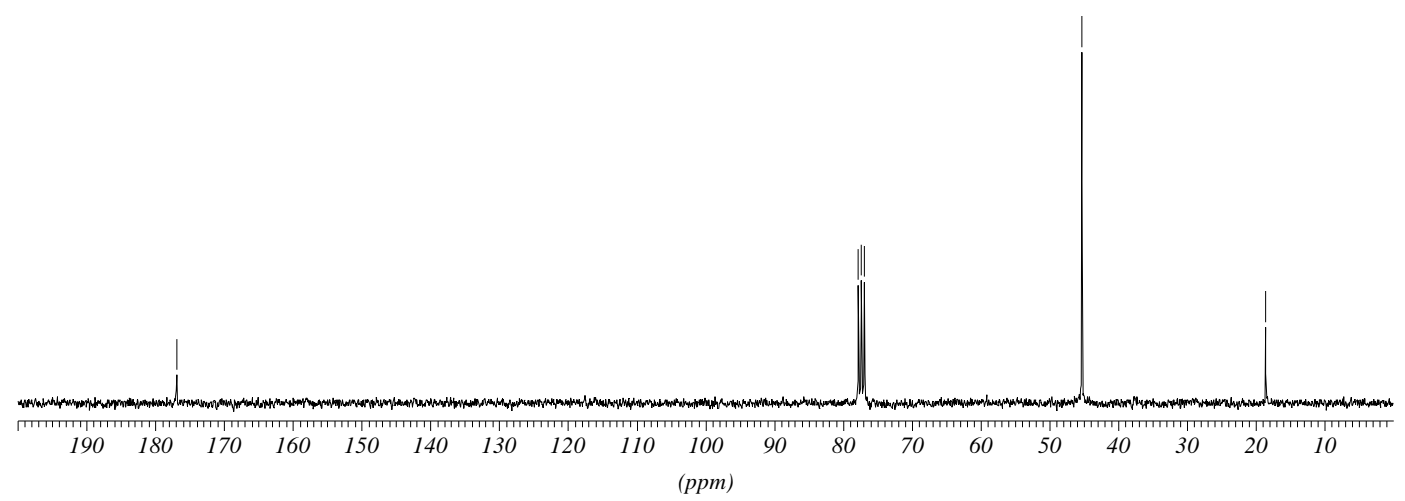



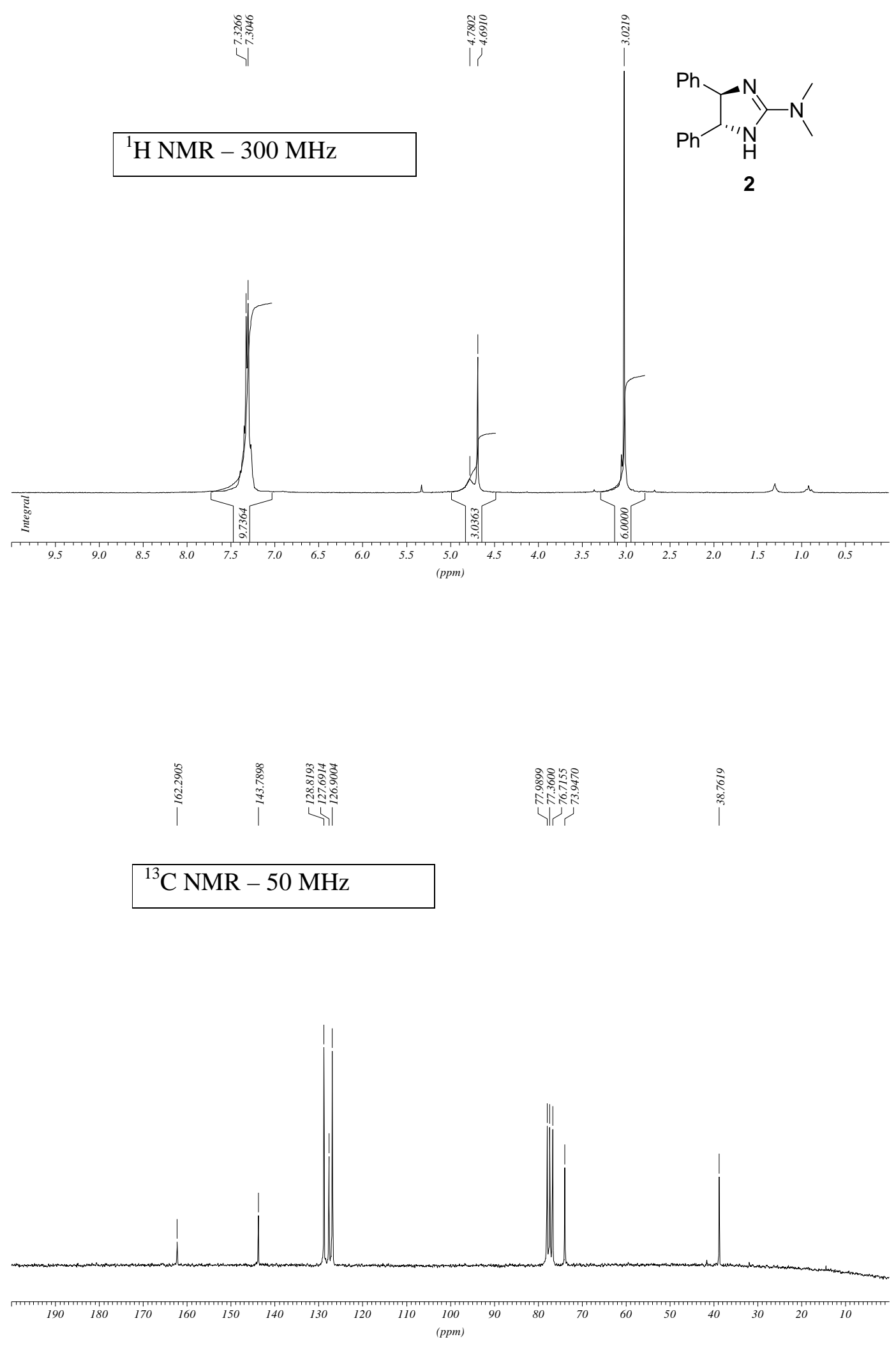
Spectral data $\left({ }^{1} \mathbf{H} /{ }^{2} \mathbf{H}\right)$

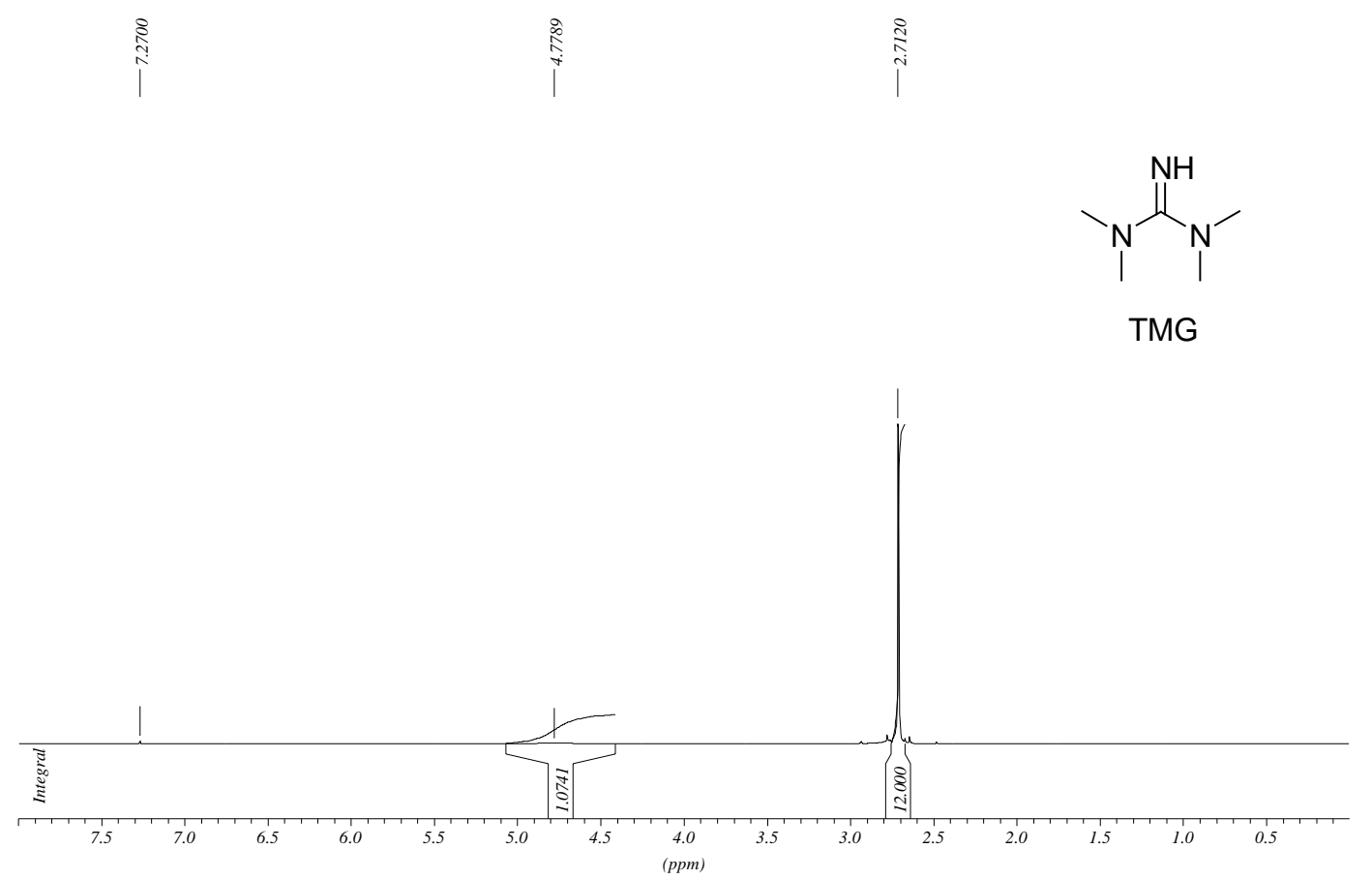



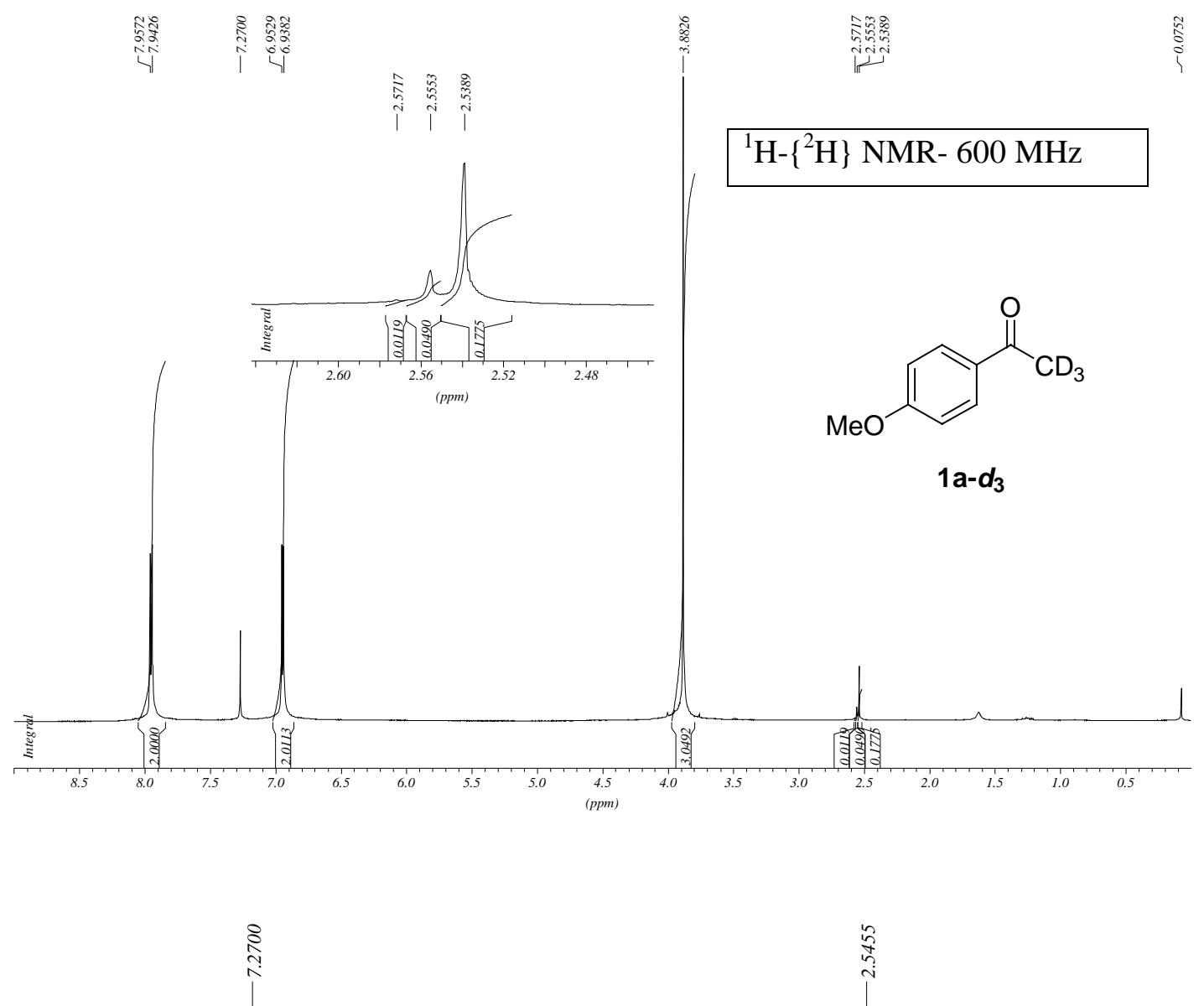

${ }^{2} \mathrm{H}$ NMR - 46.07 MHz

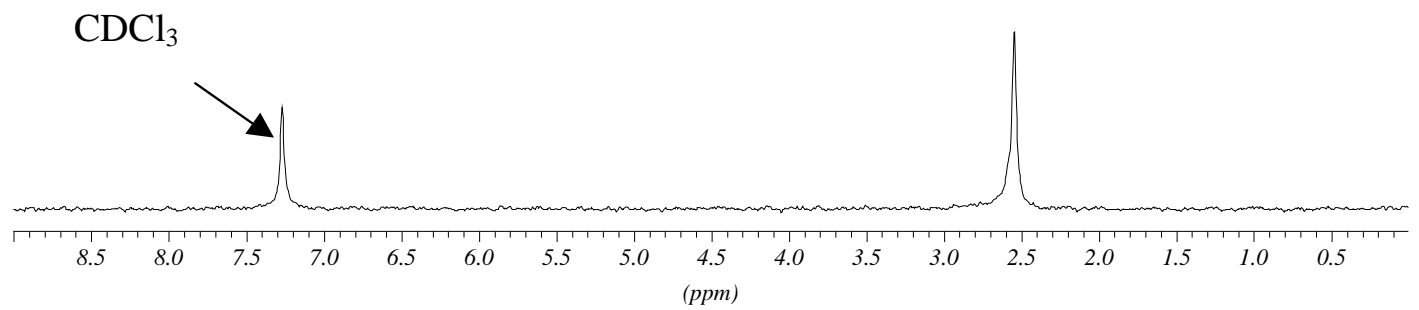




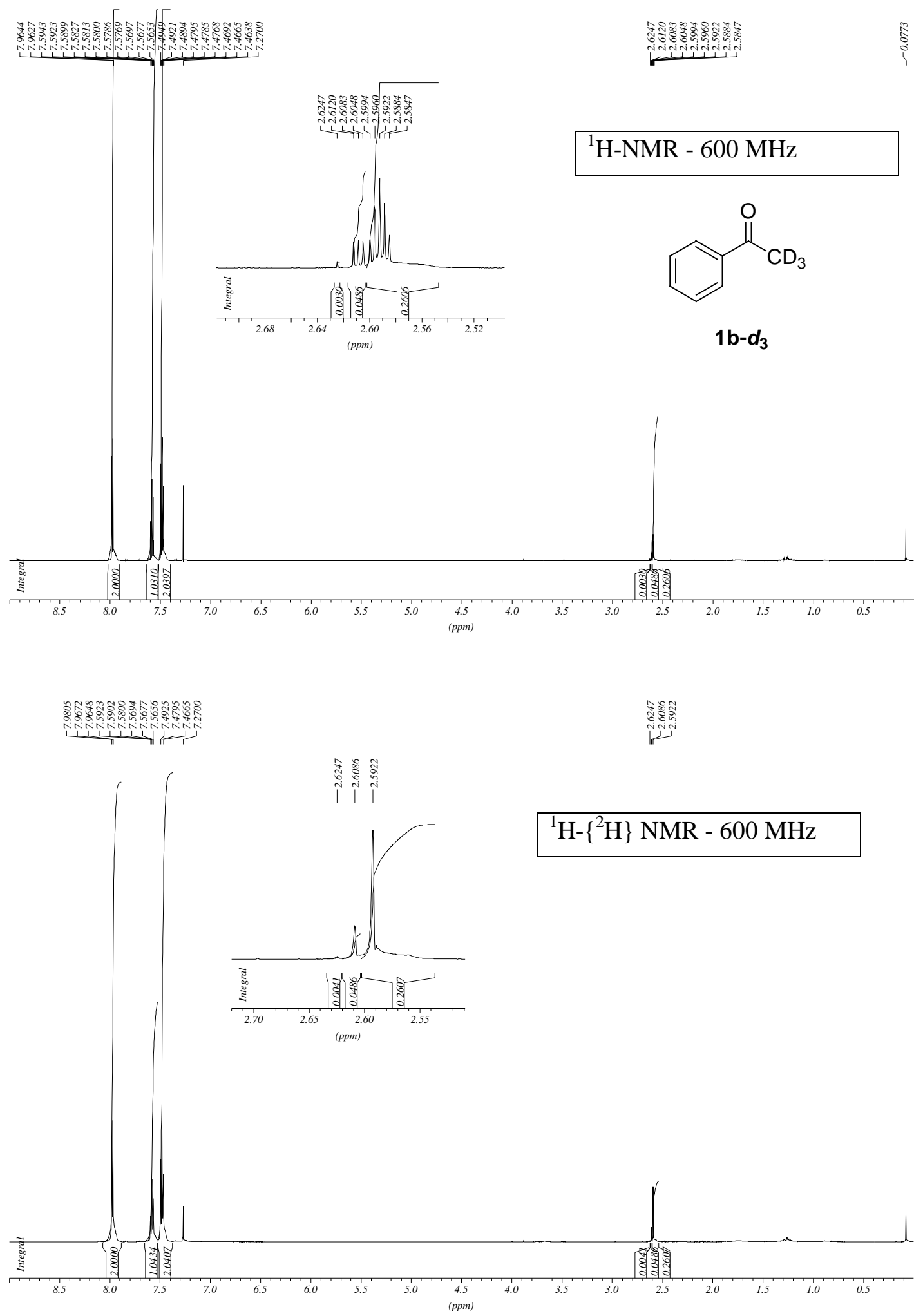


$\stackrel{8}{i}$

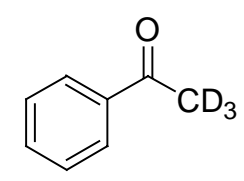

$1 b-d_{3}$ à

${ }^{2} \mathrm{H}$ NMR - $46 \mathrm{MHz}$

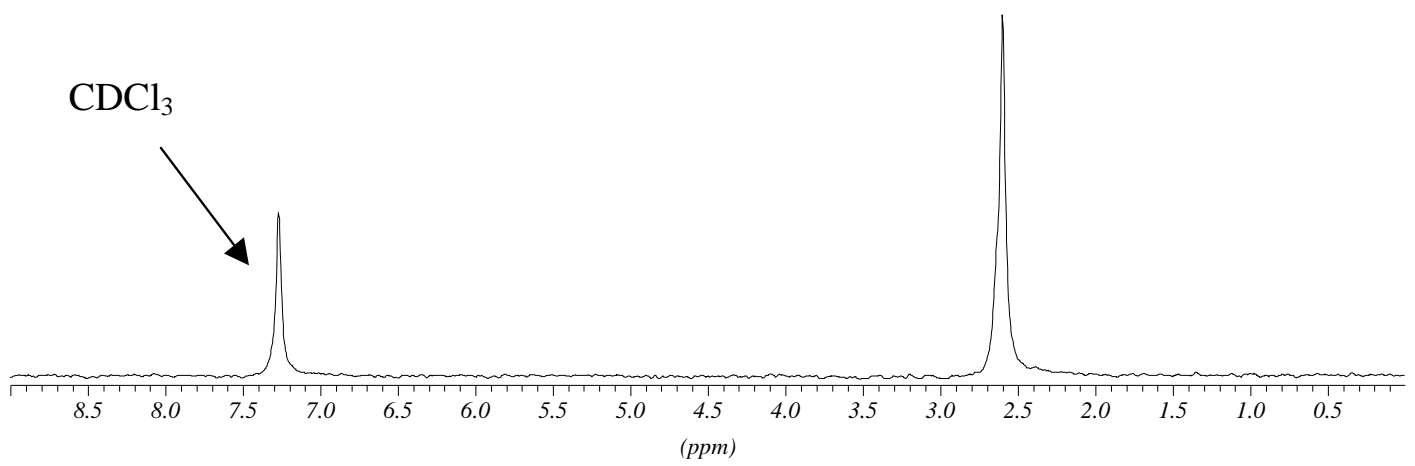


$\underline{\text { GC }}$

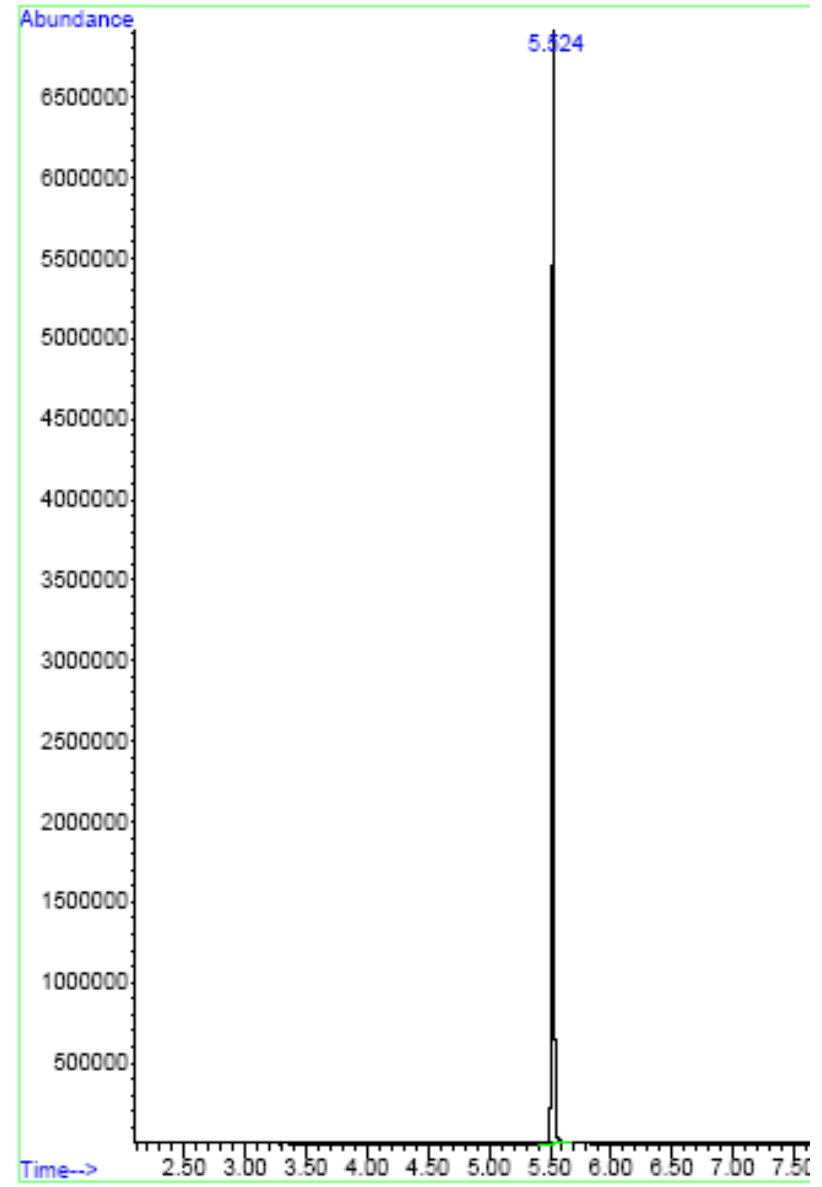<smiles>CC(=O)c1ccccc1</smiles>

$1 b$

\section{$\underline{\text { MS }}$}

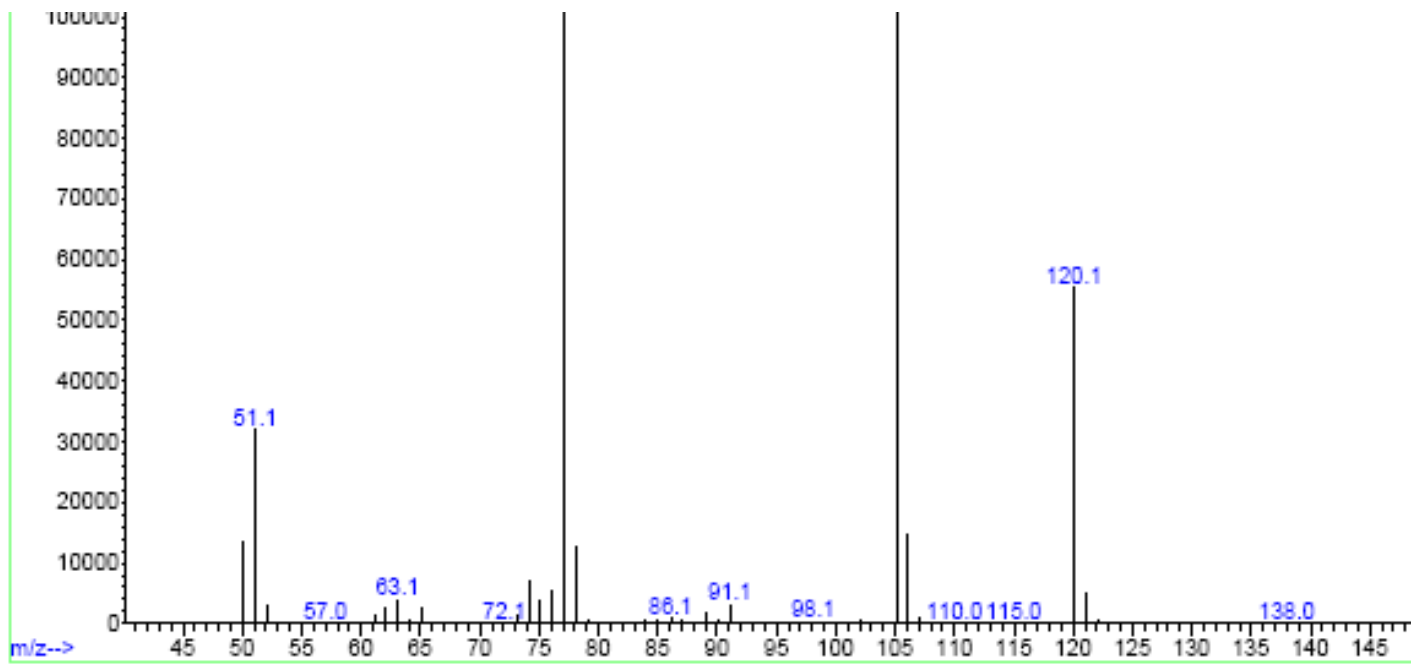


$\underline{\text { GC }}$
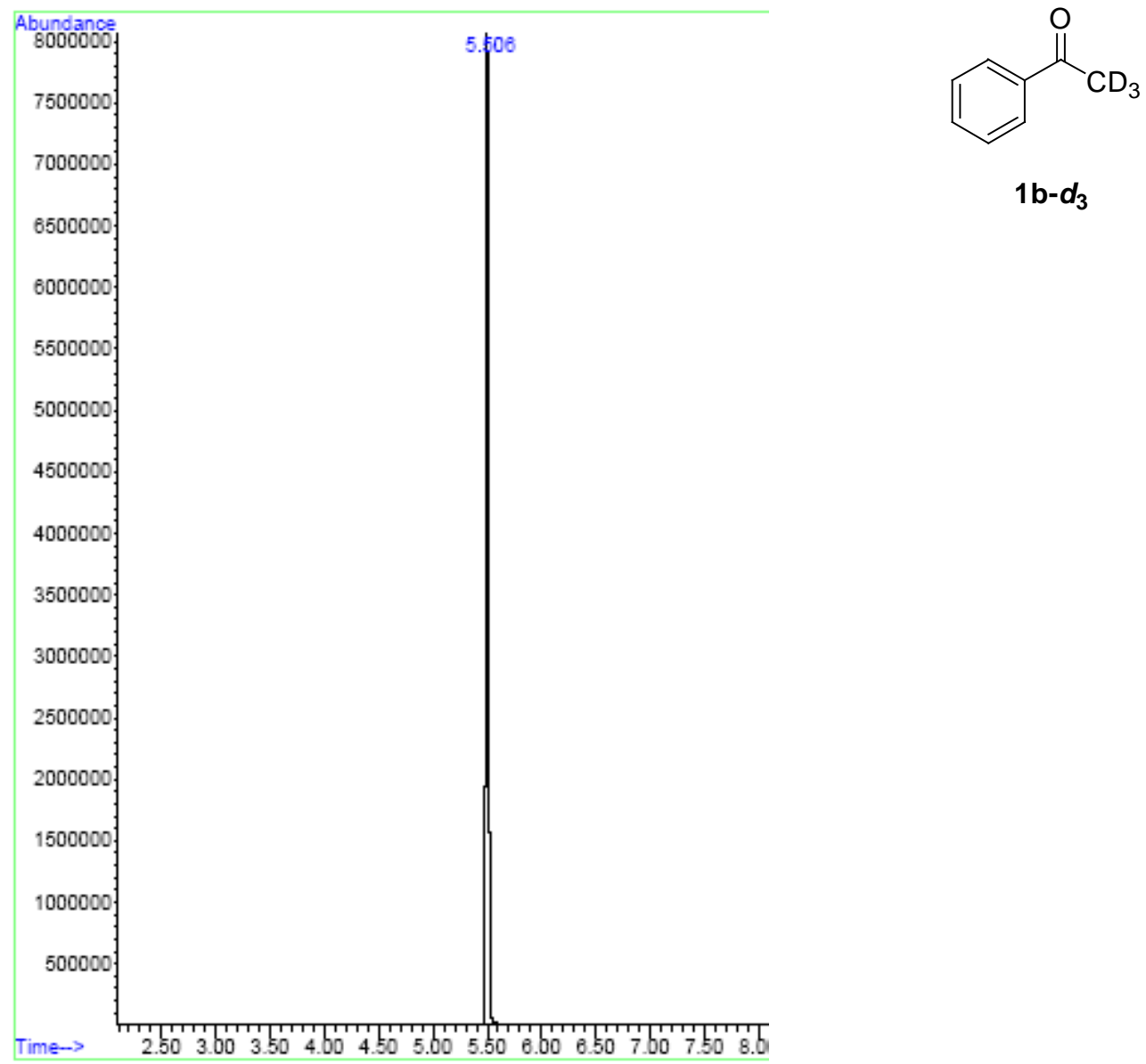

$\underline{\text { MS }}$

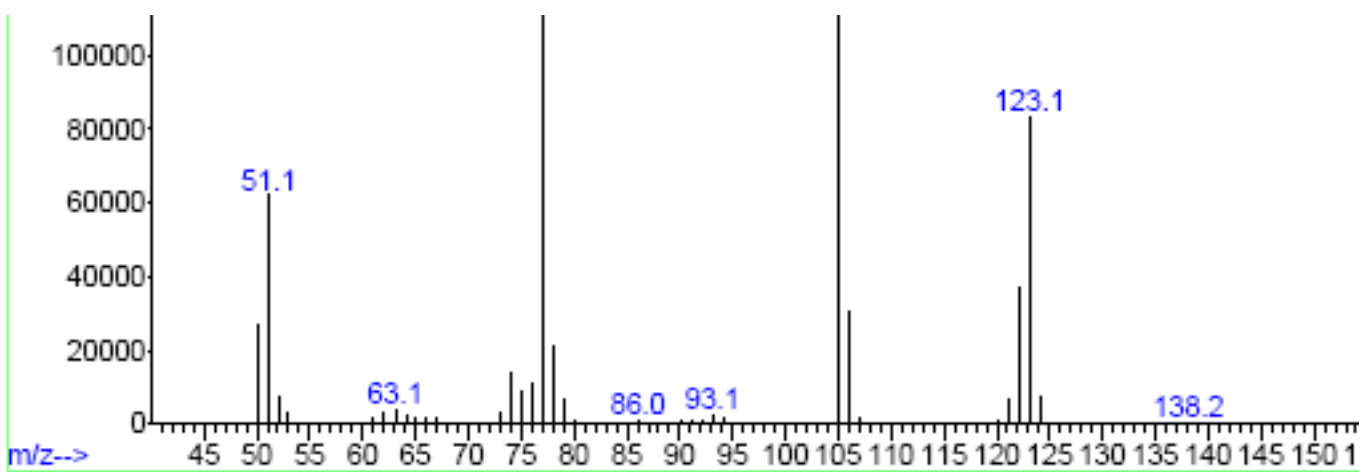




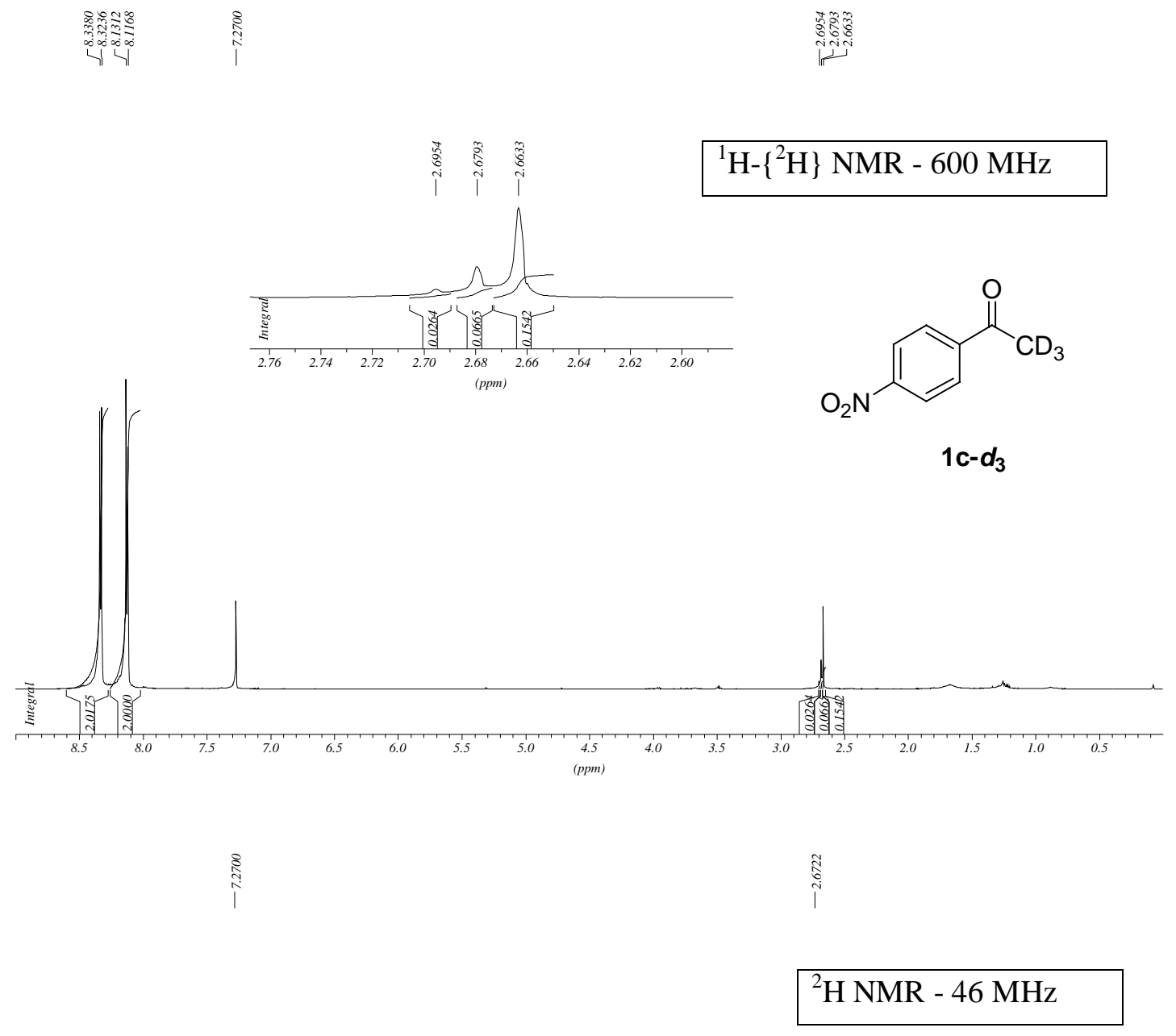

\section{$\mathrm{CDCl}_{3}$}

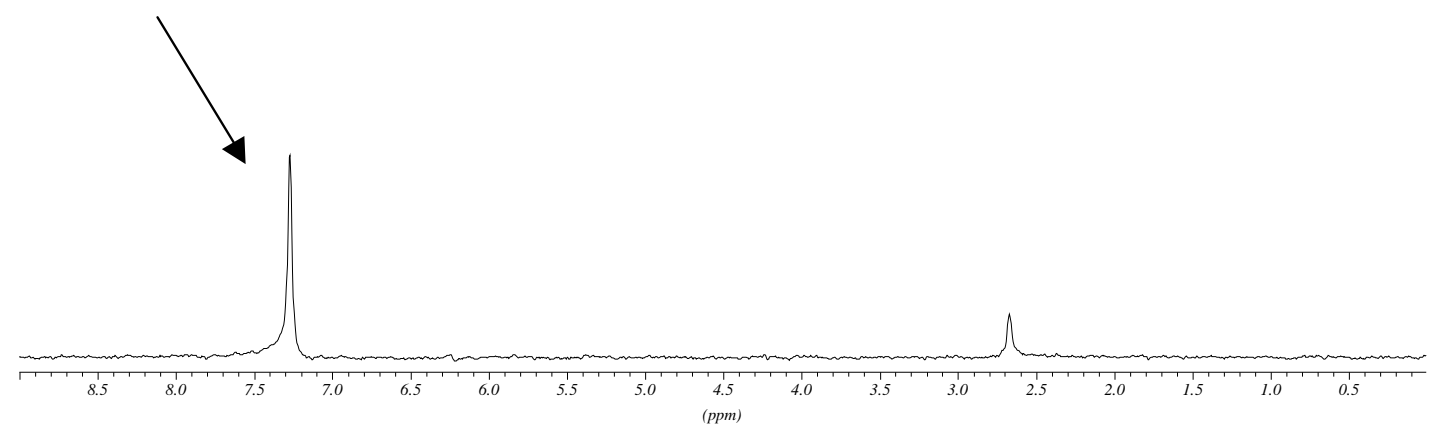




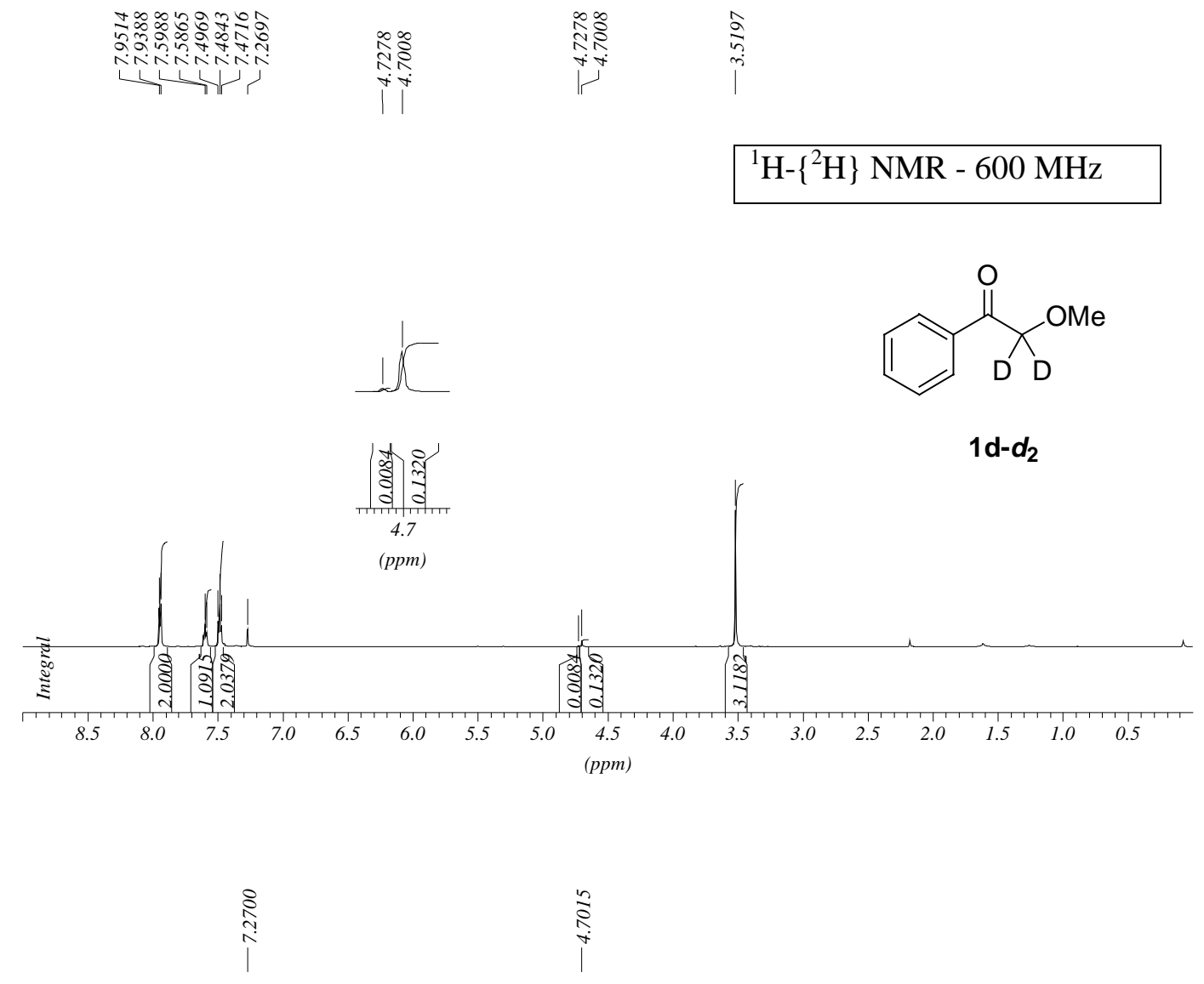

${ }^{2} \mathrm{H}$ NMR - $46 \mathrm{MHz}$

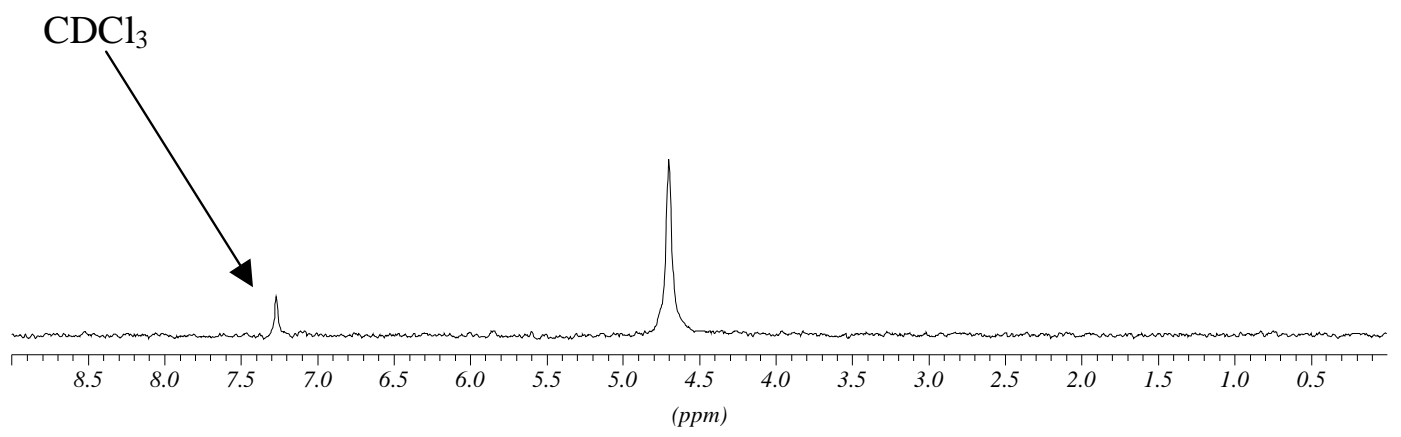



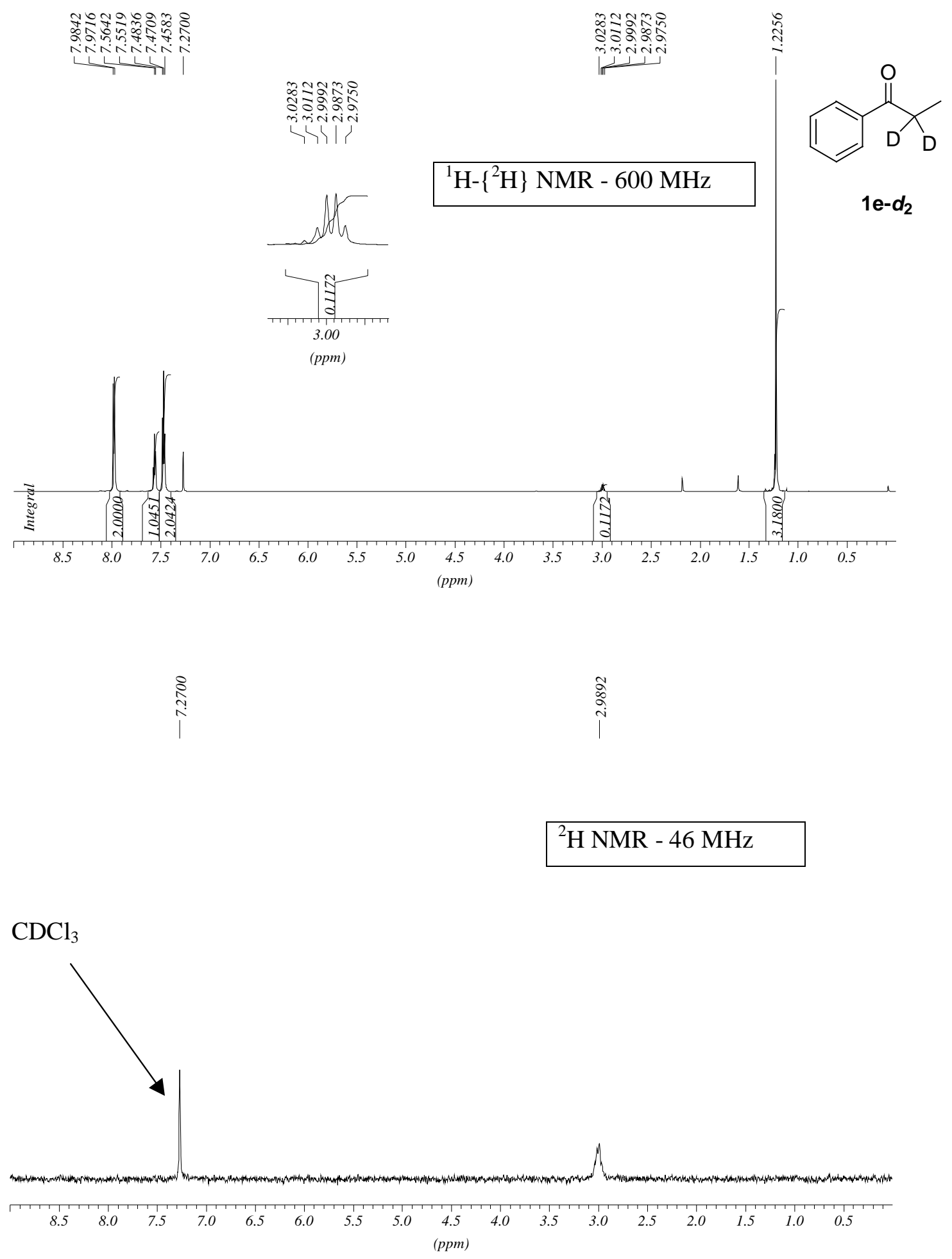


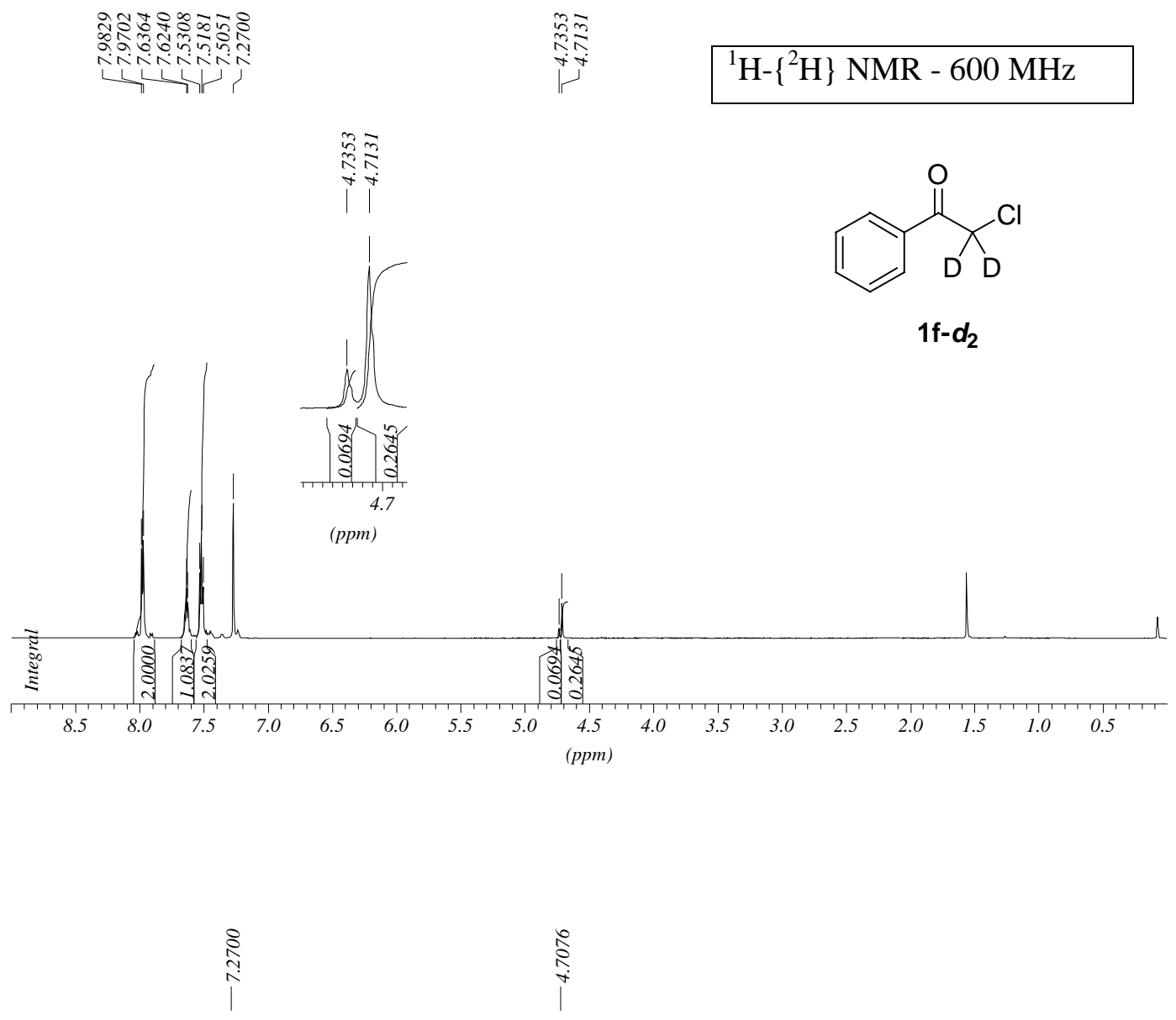

\section{${ }^{2} \mathrm{H}$ NMR - $46 \mathrm{MHz}$}

\section{$\mathrm{CDCl}_{3}$}
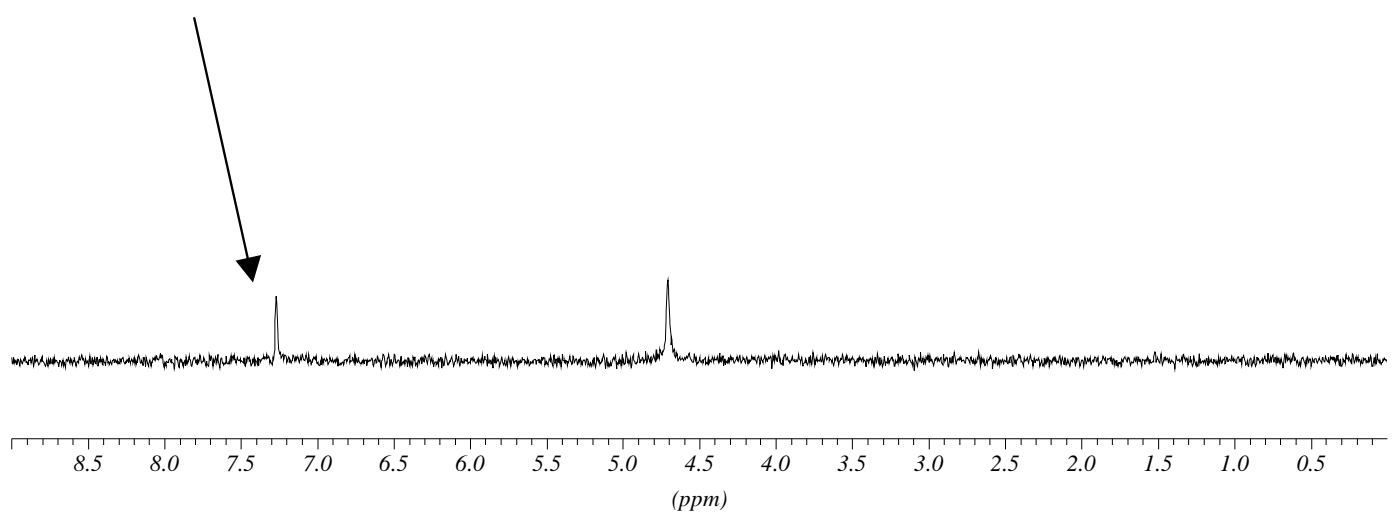

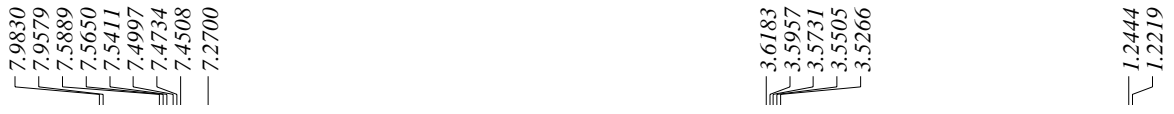

${ }^{1} \mathrm{H}-\left\{{ }^{2} \mathrm{H}\right\}$ NMR $-600 \mathrm{MHz}$<smiles>[2H]C(C)(C)C(=O)c1ccccc1</smiles>

$1 \mathrm{~g}-d_{1}$

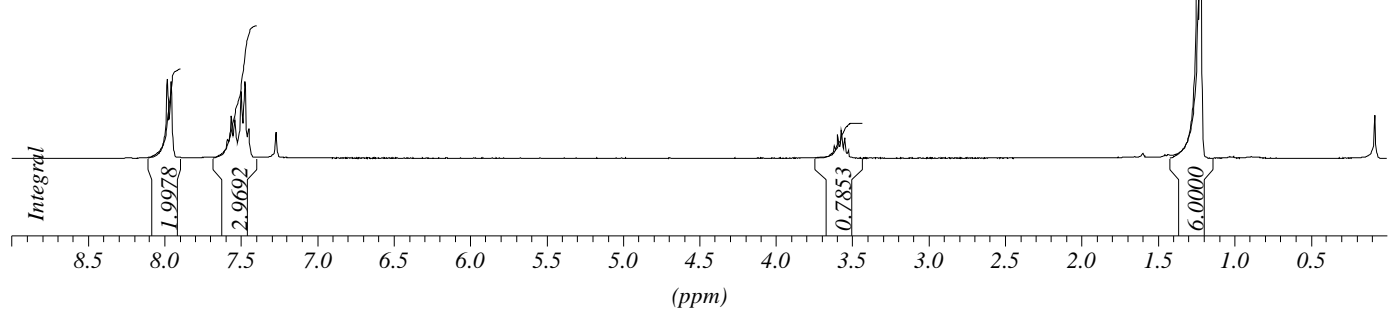

$\stackrel{8}{\stackrel{2}{i}}$

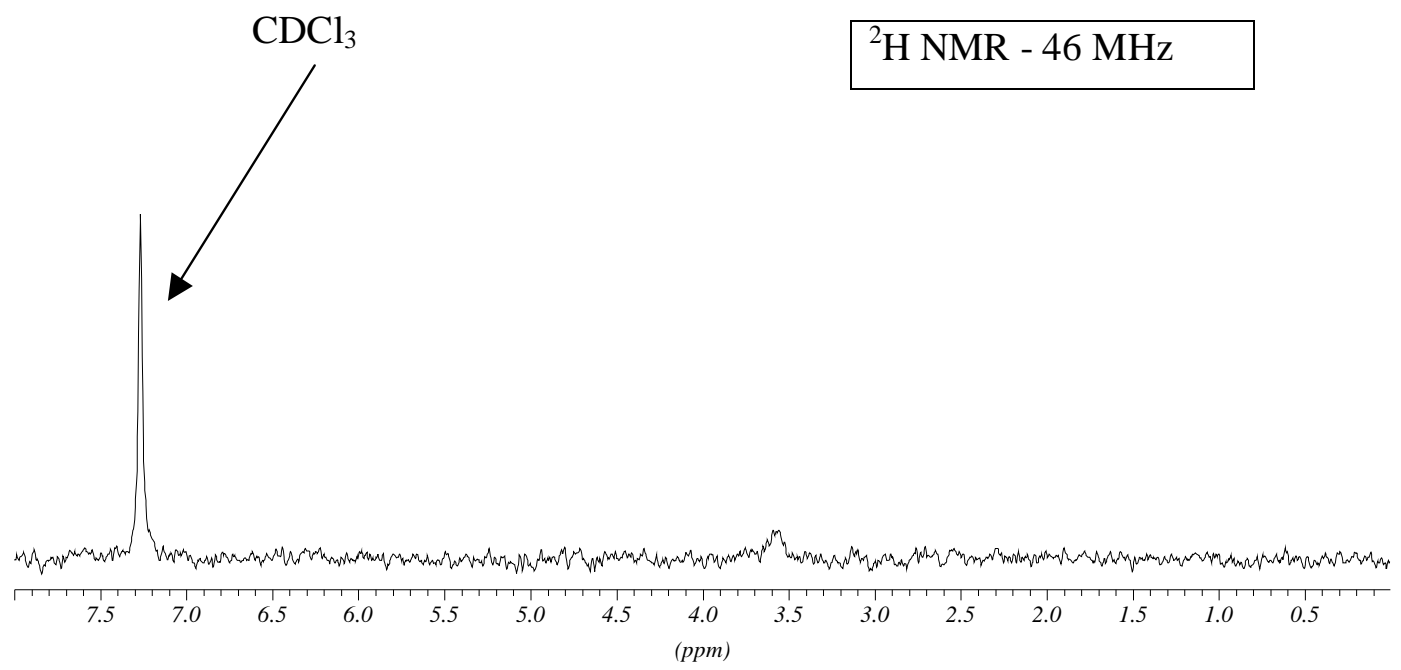




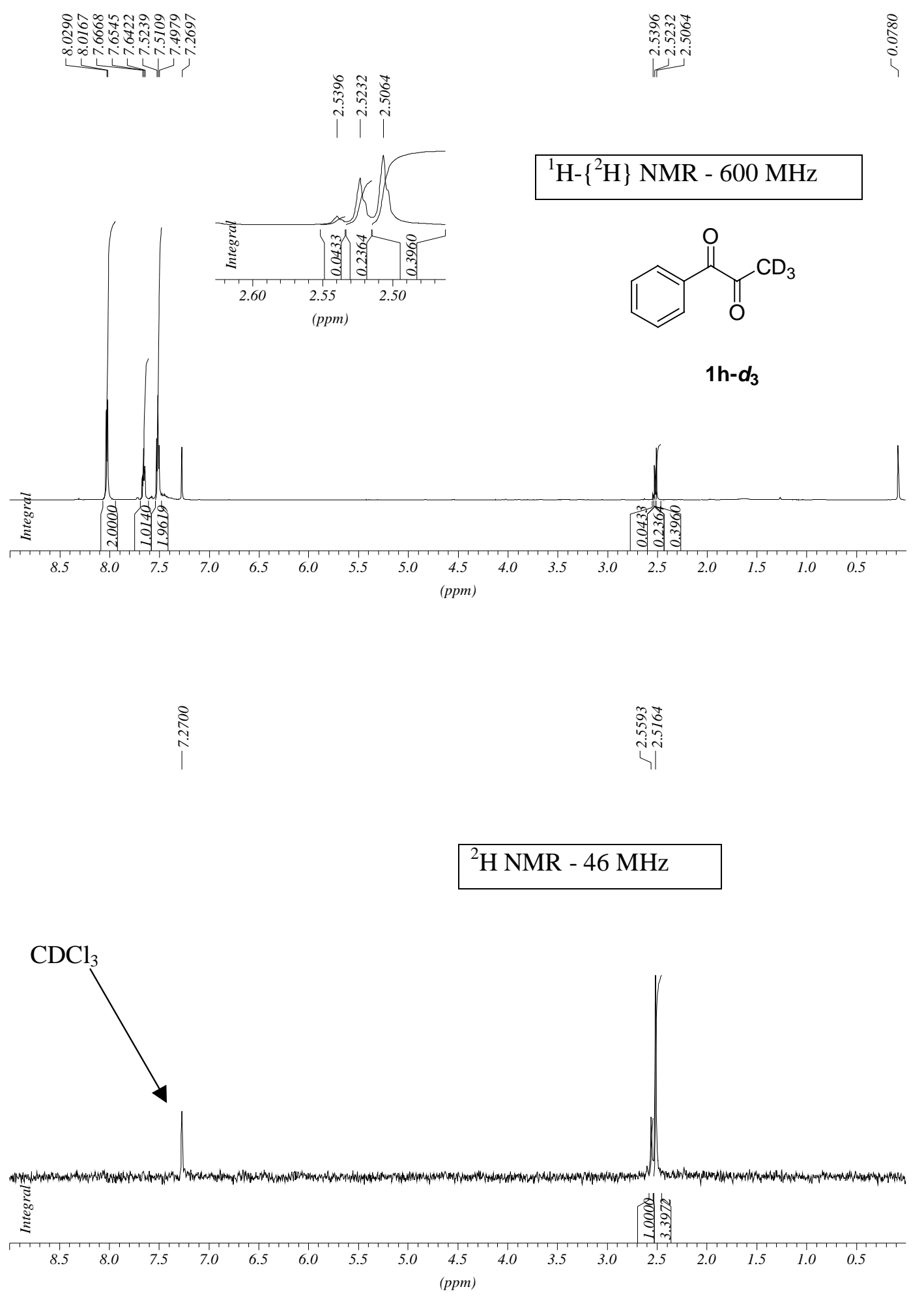



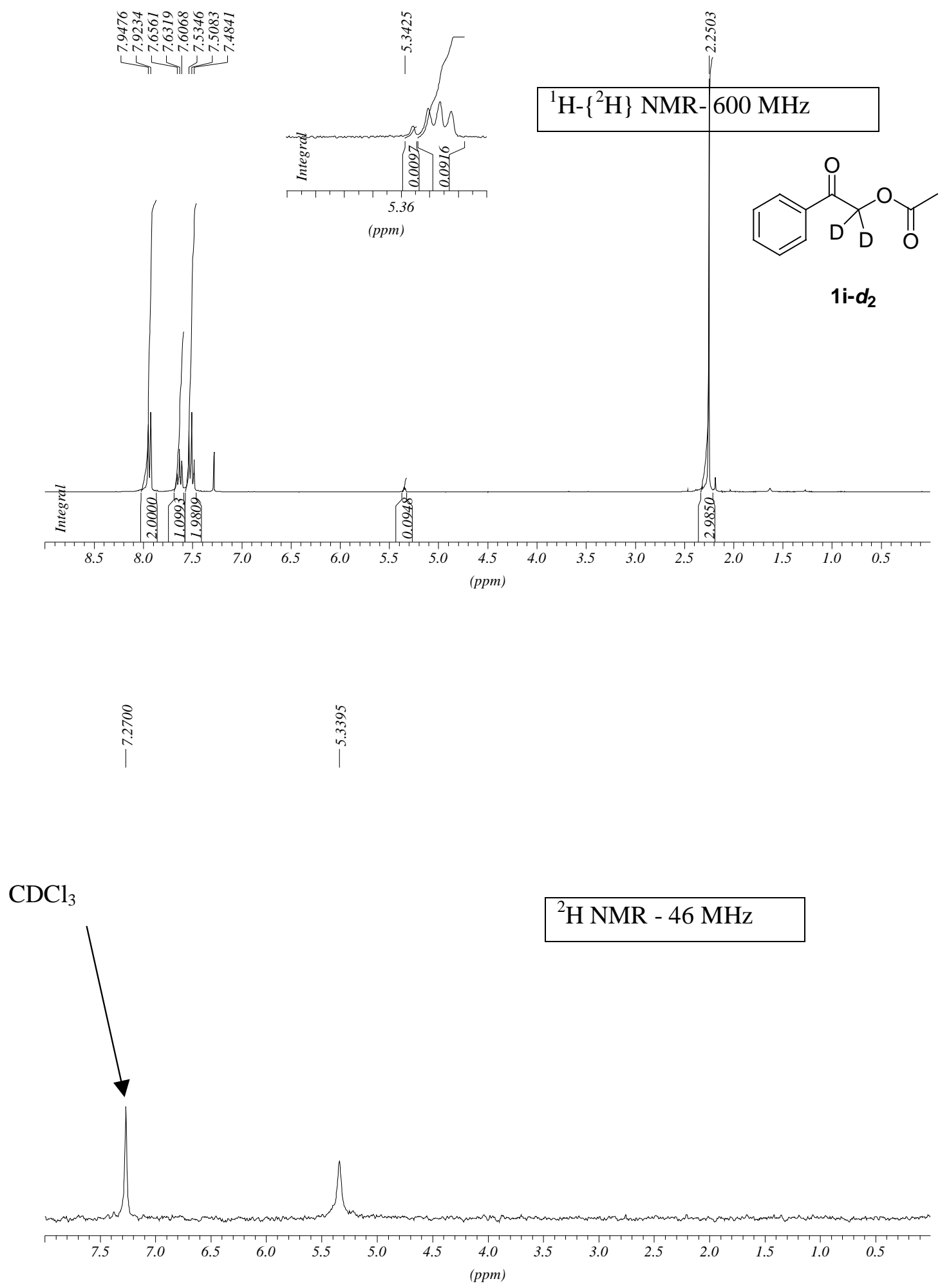


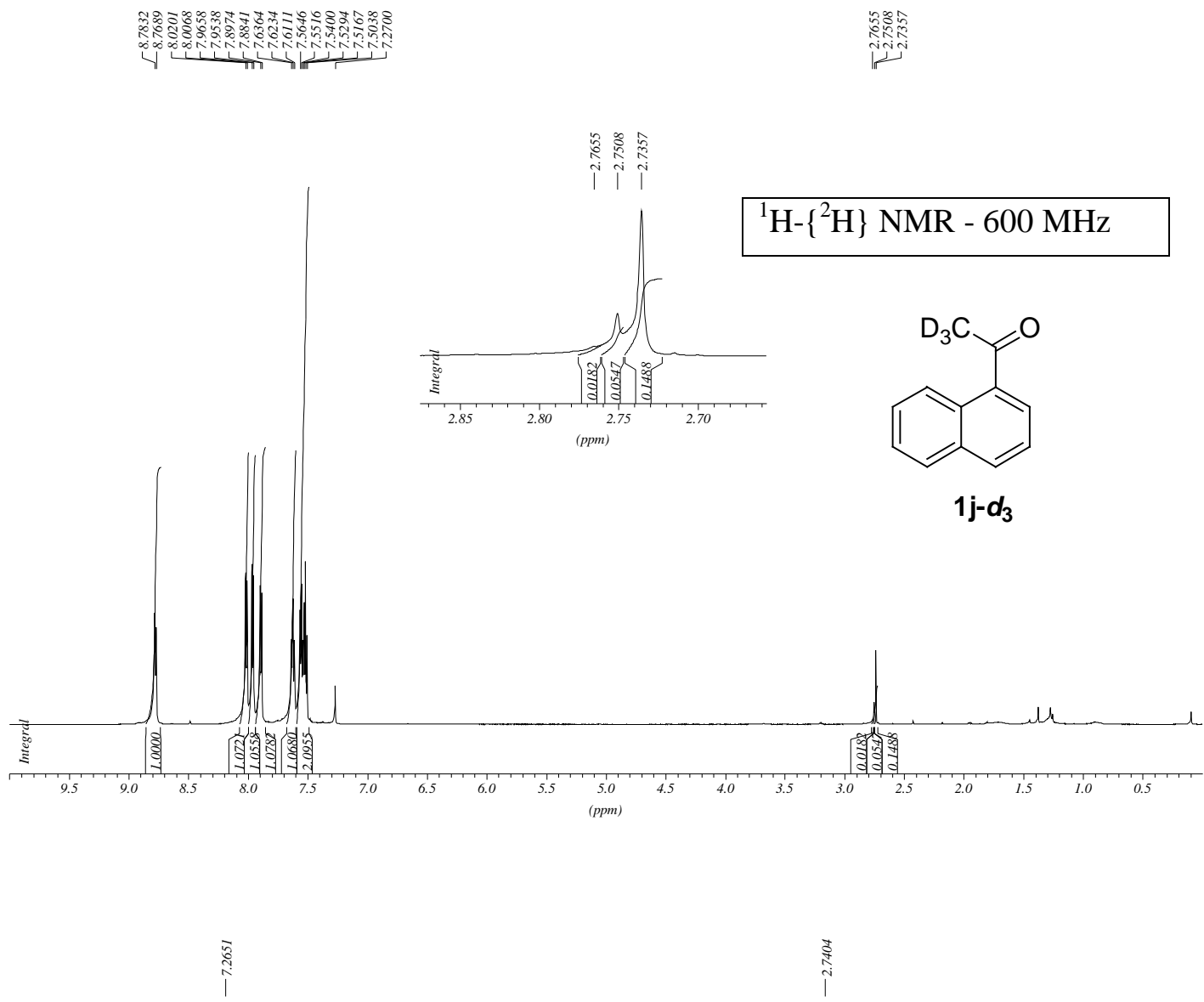

${ }^{2} \mathrm{H} \mathrm{NMR}-46 \mathrm{MHz}$

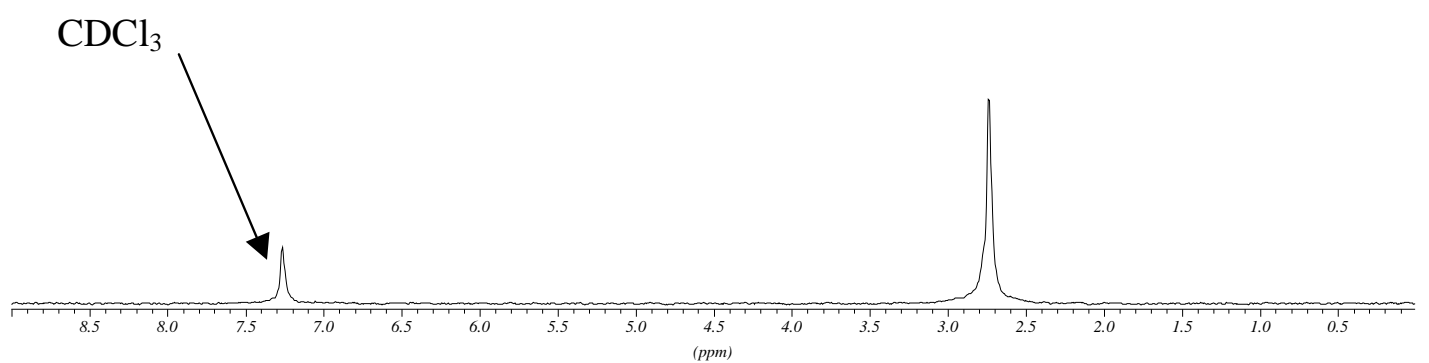



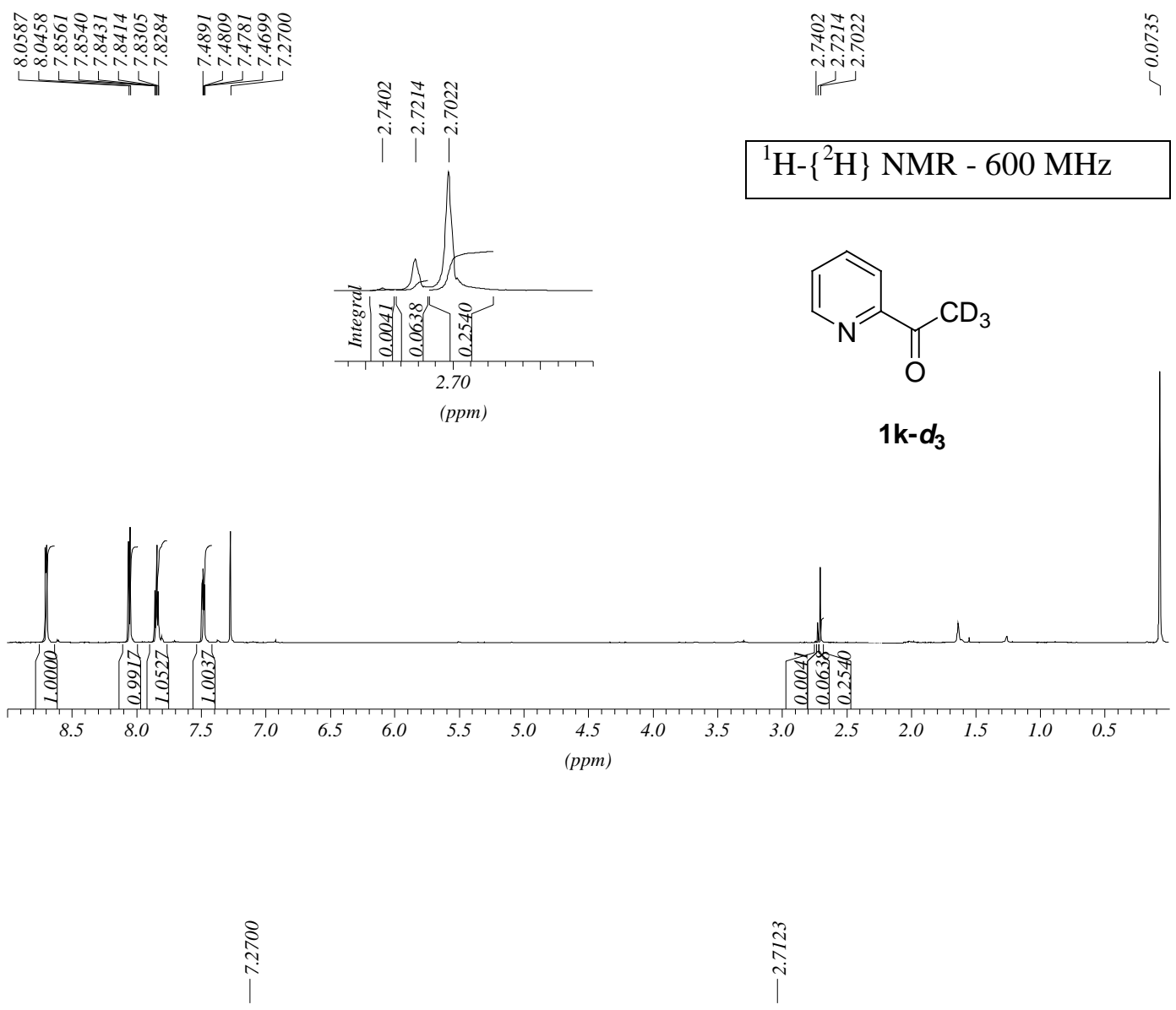

${ }^{2} \mathrm{H}$ NMR - $46 \mathrm{MHz}$

\section{$\mathrm{CDCl}_{3}$}

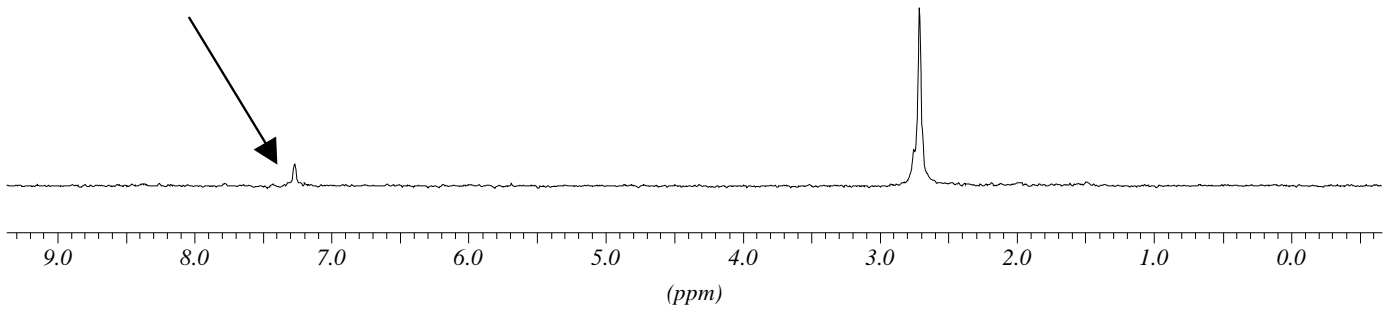




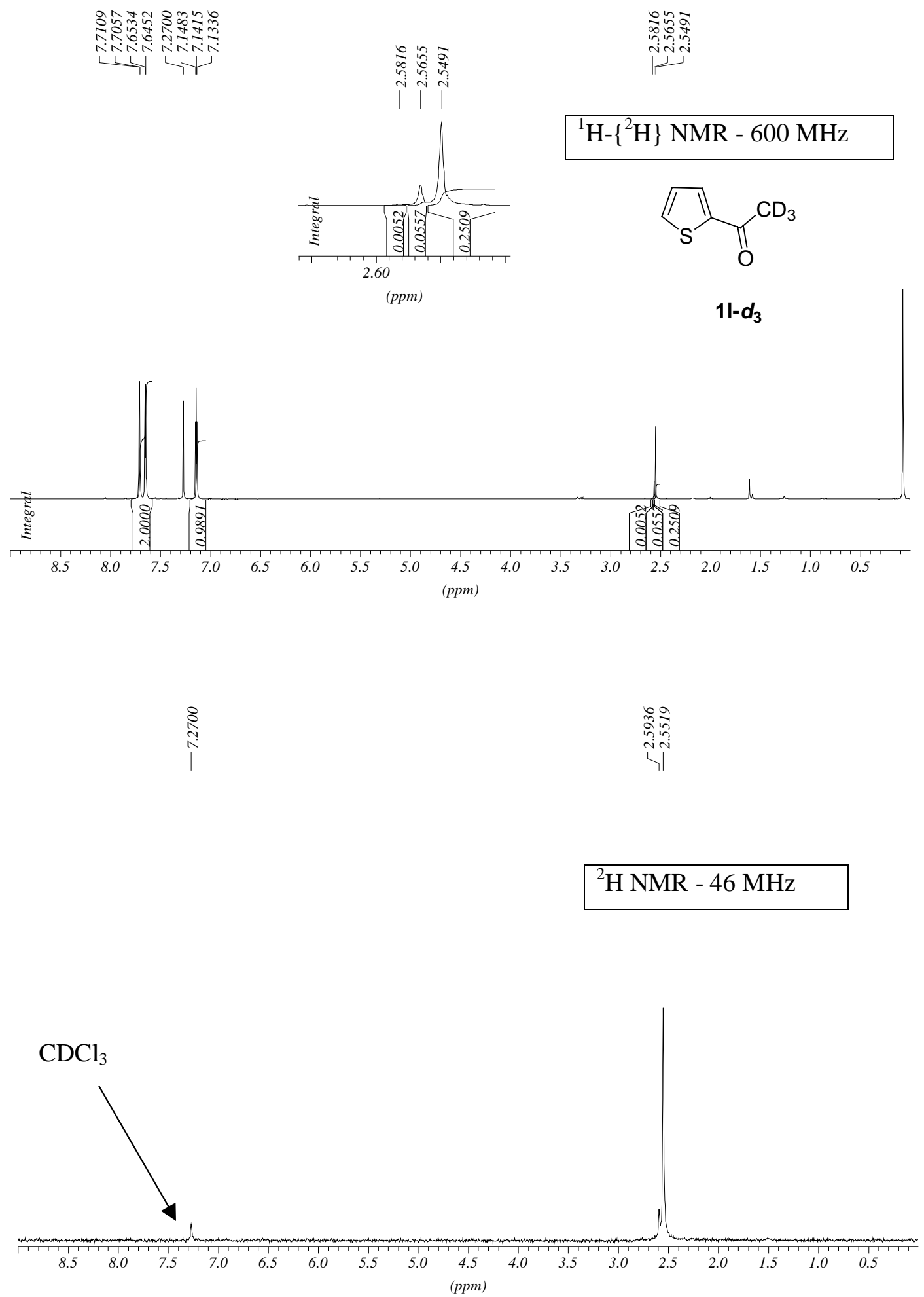



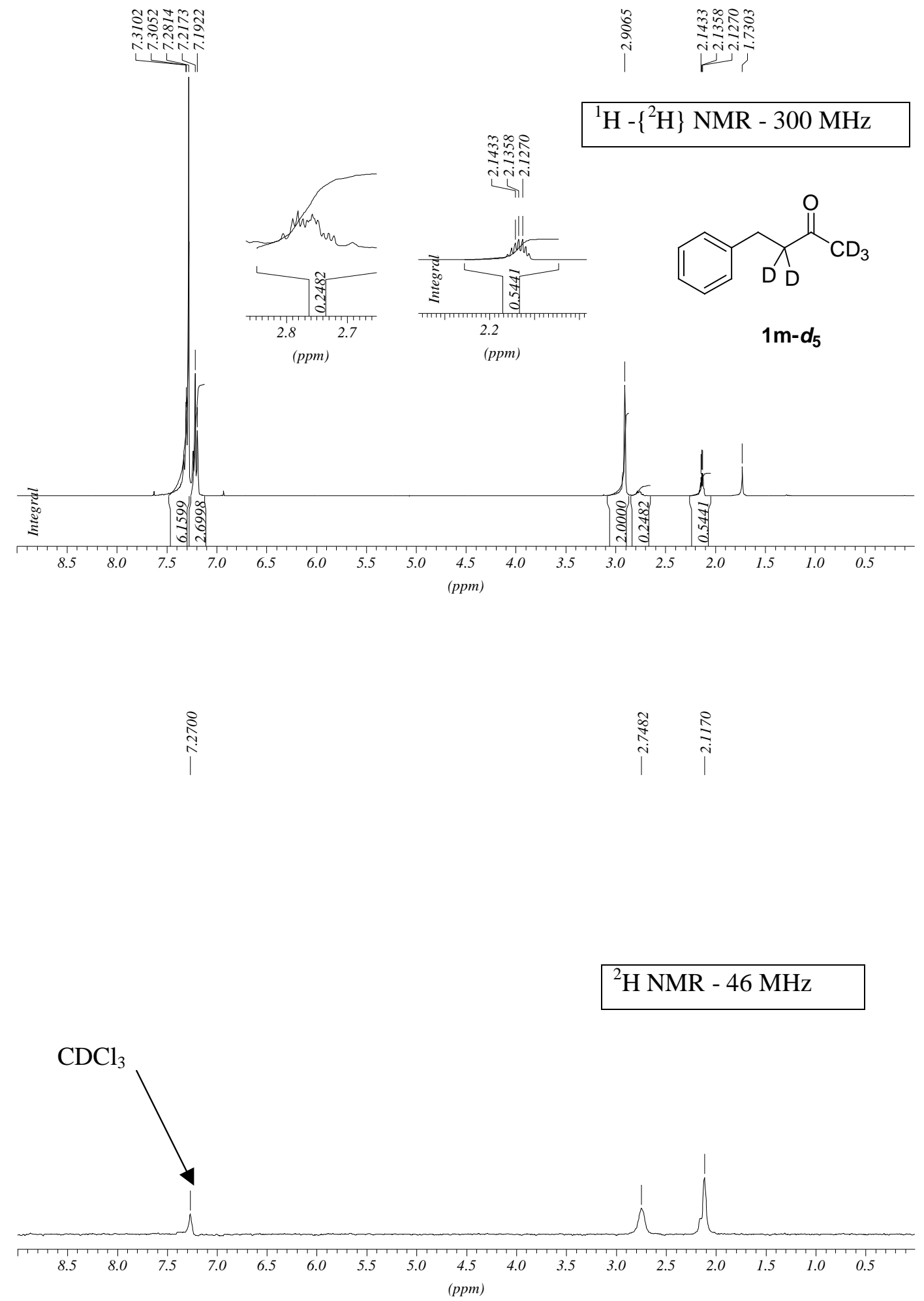
$\underline{\text { GC }}$

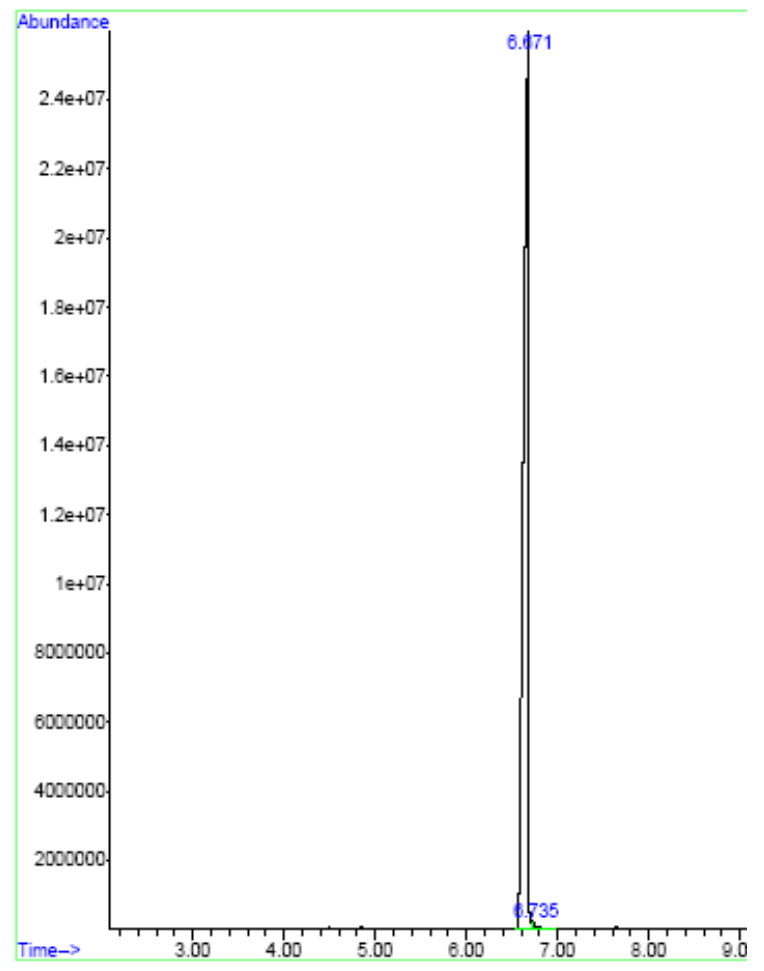

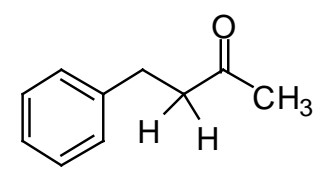

$1 \mathrm{~m}$

\section{$\underline{\text { MS }}$}

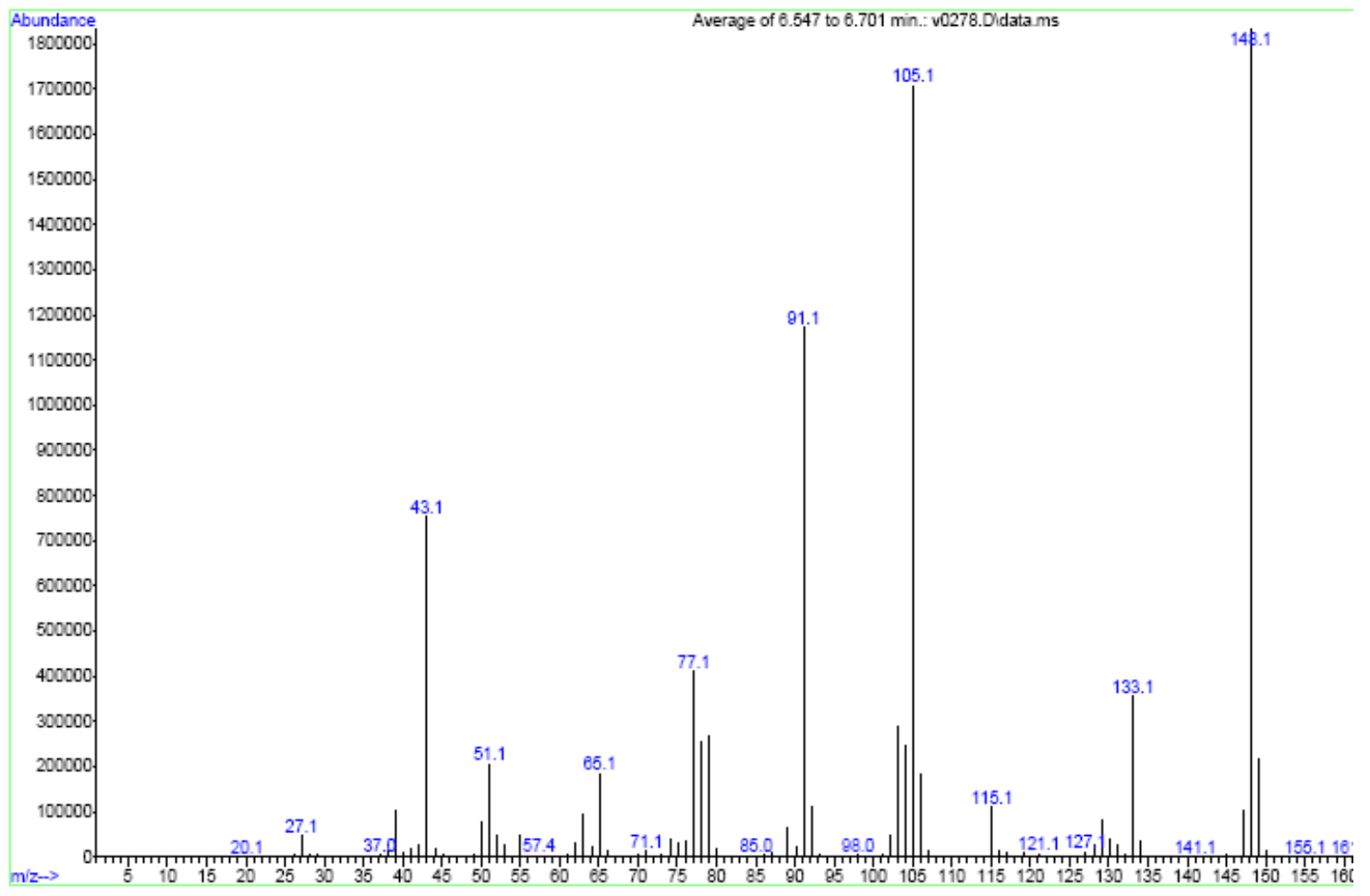




\section{$\underline{\text { GC }}$}

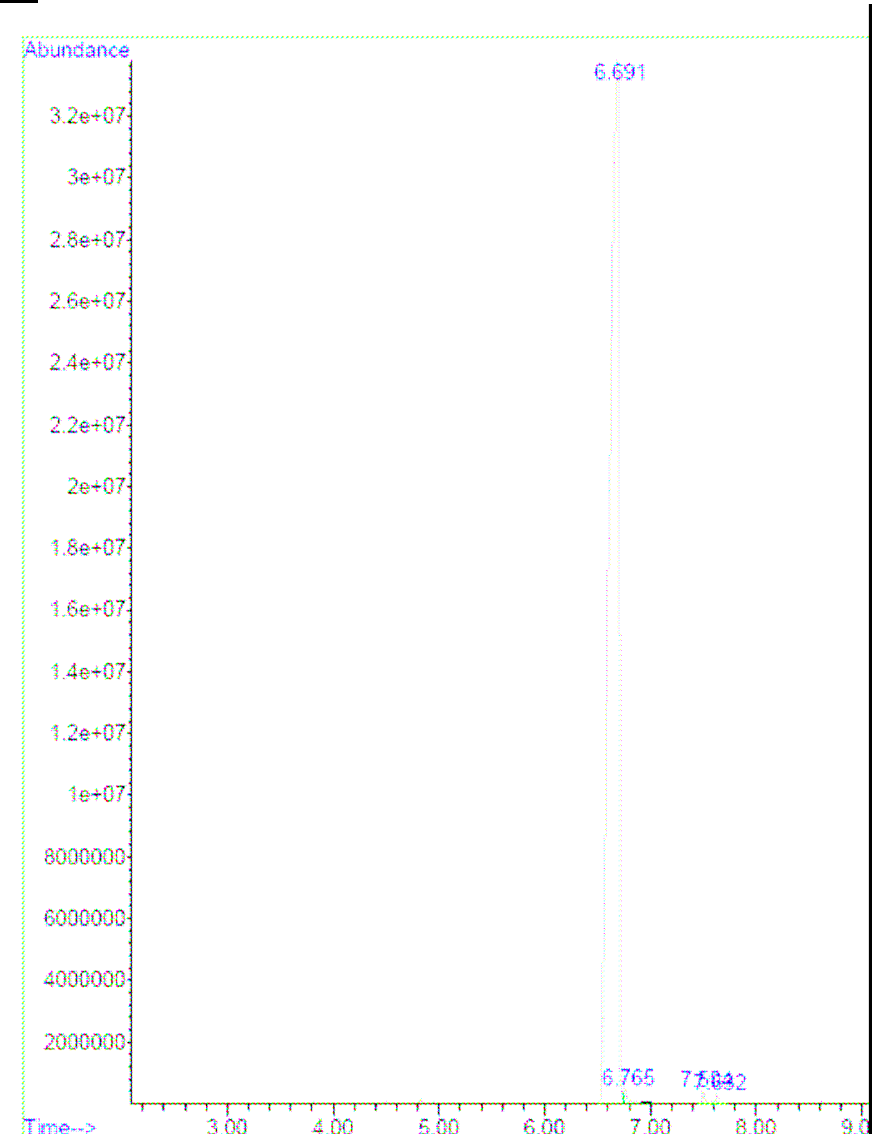

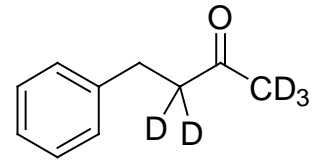

$1 \mathrm{~m}-d_{5}$

\section{$\underline{\text { MS }}$}

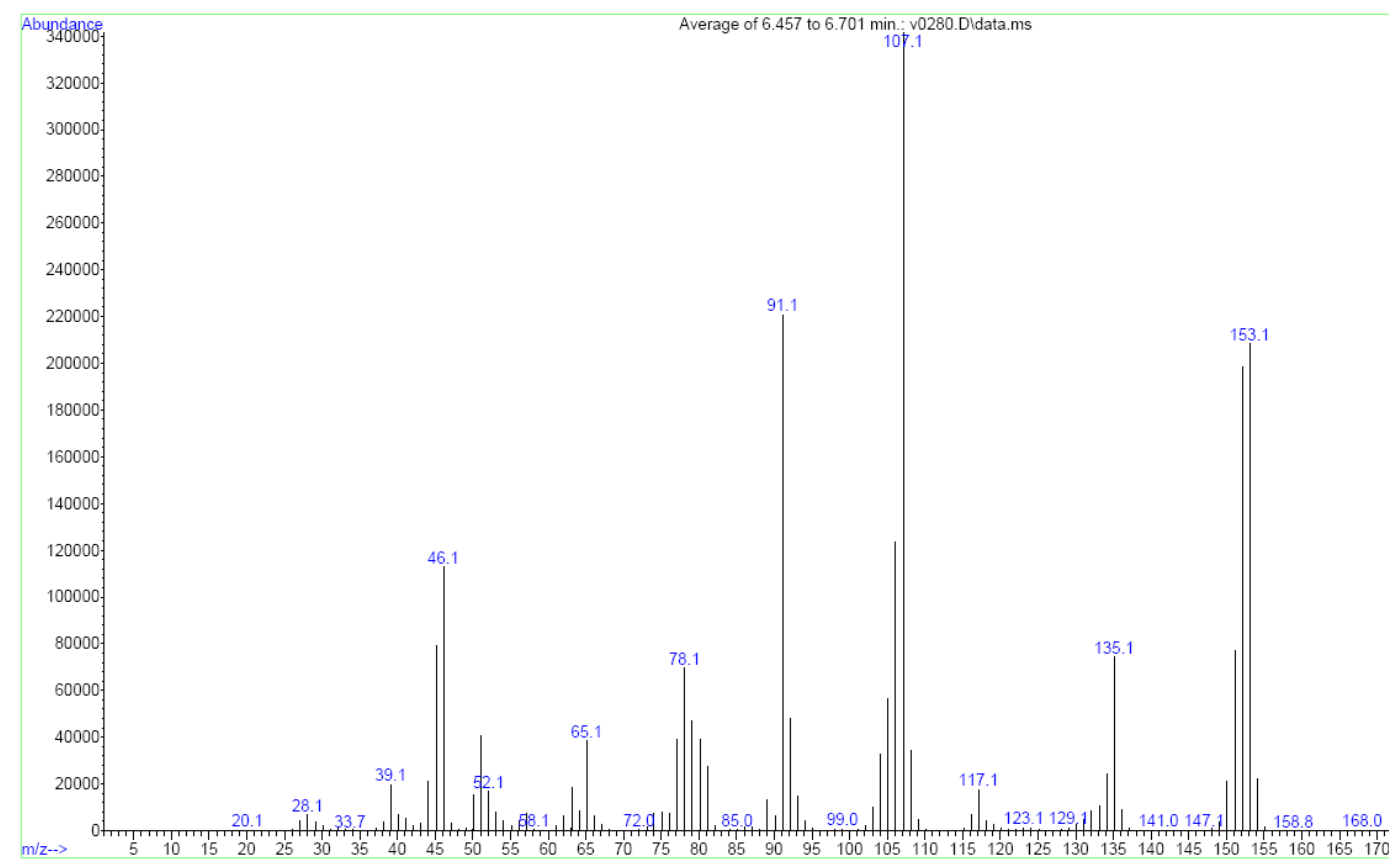




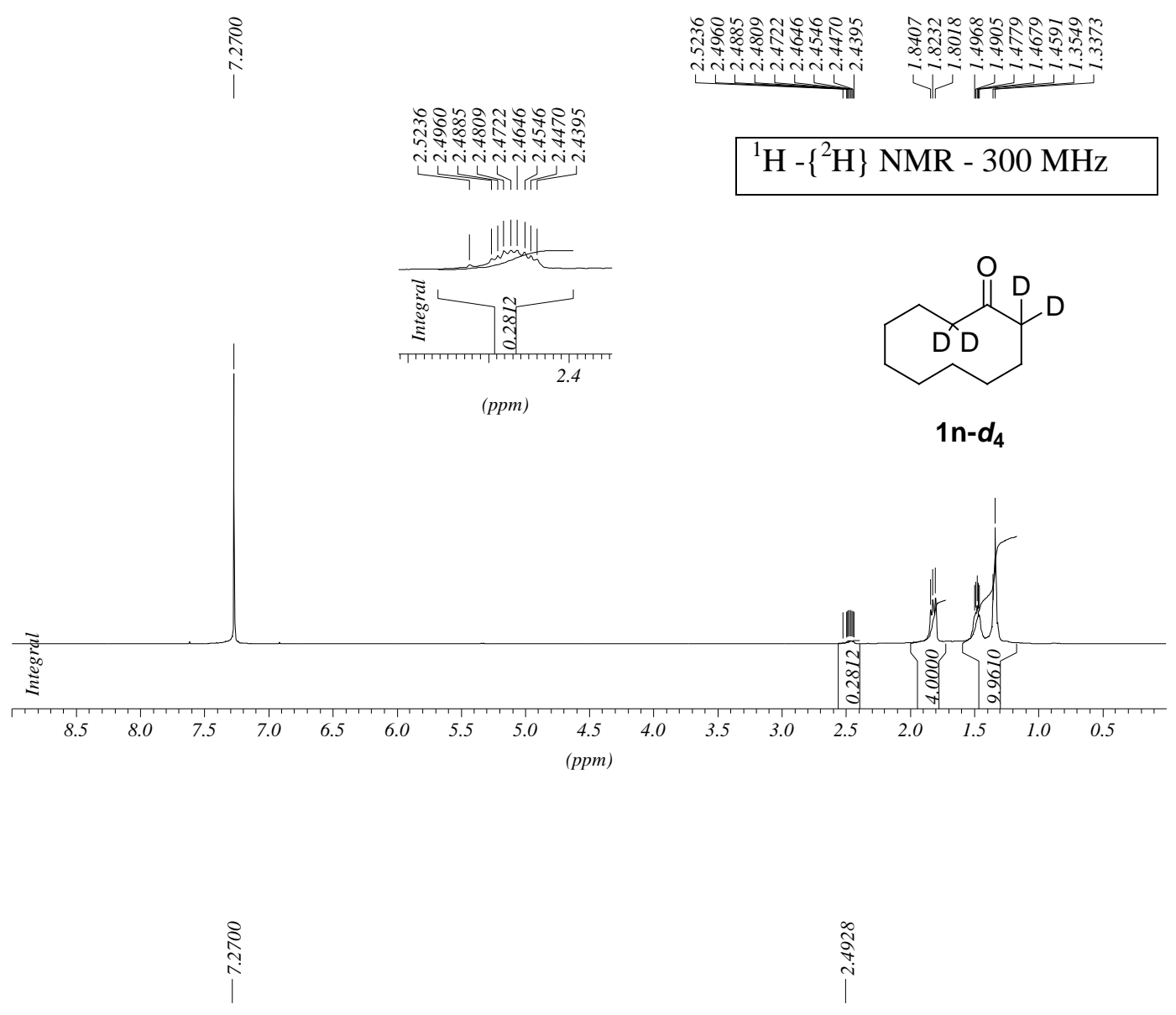

\section{${ }^{2} \mathrm{H}$ NMR - $46 \mathrm{MHz}$}

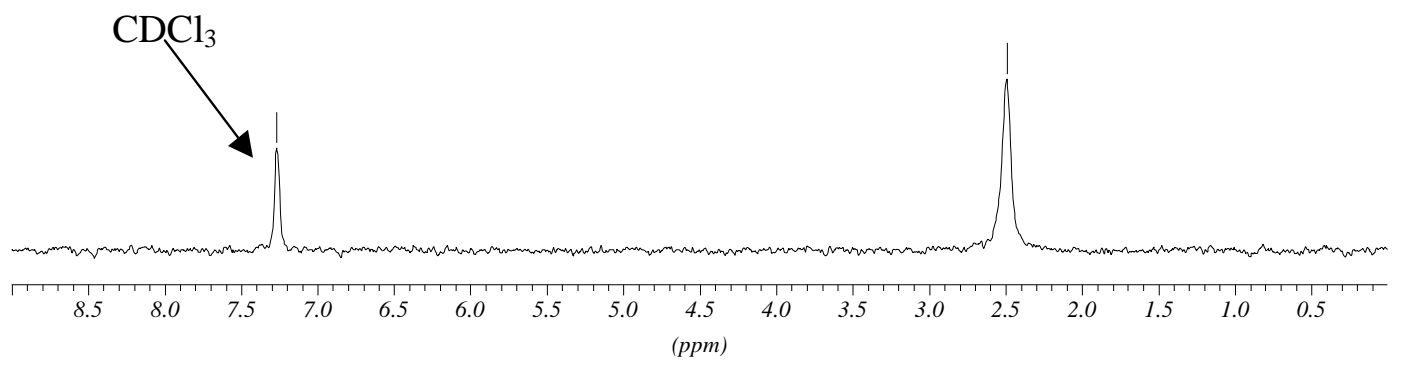


GC

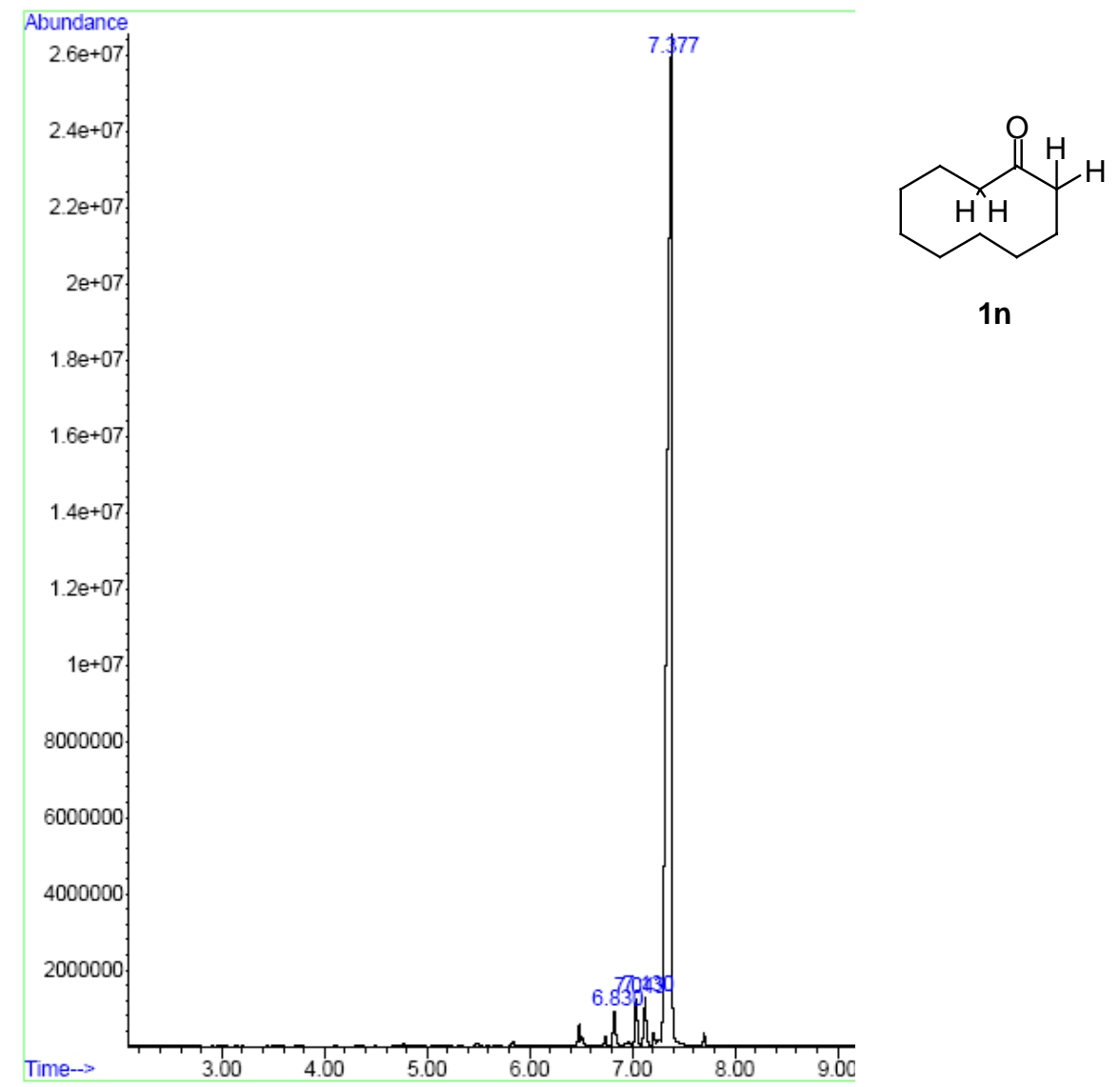

\section{$\underline{\text { MS }}$}

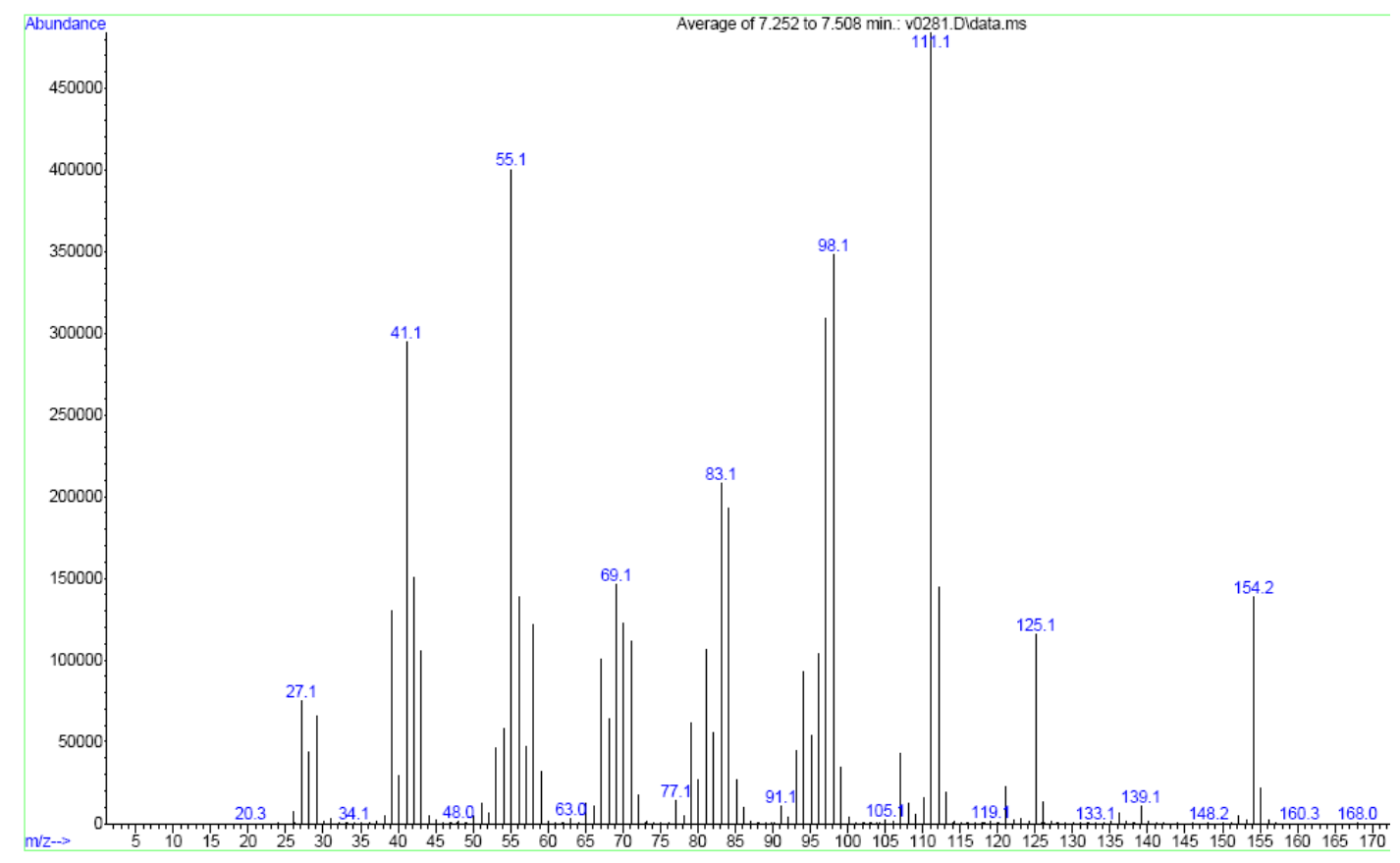




\section{GC}
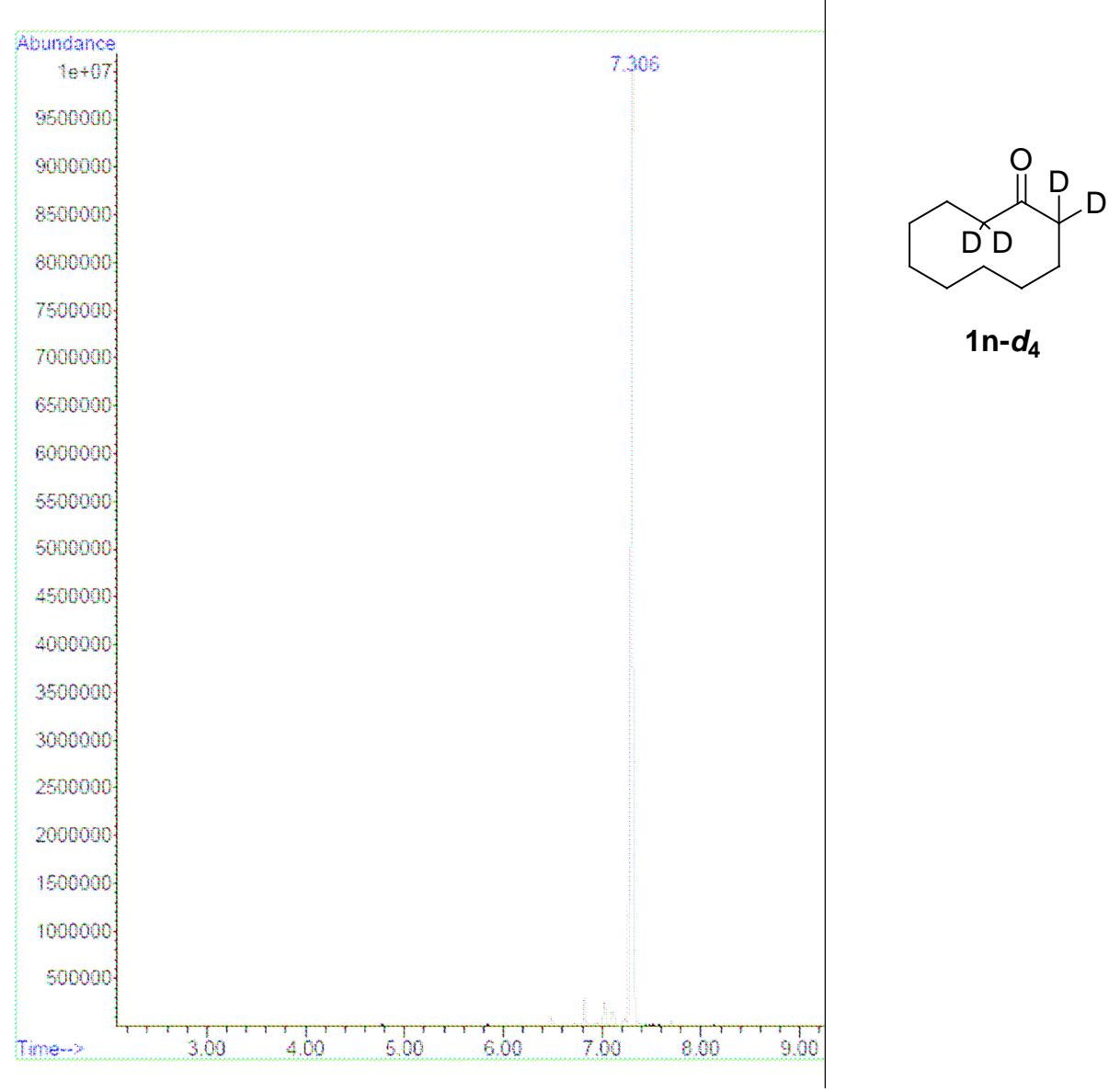

\section{MS}

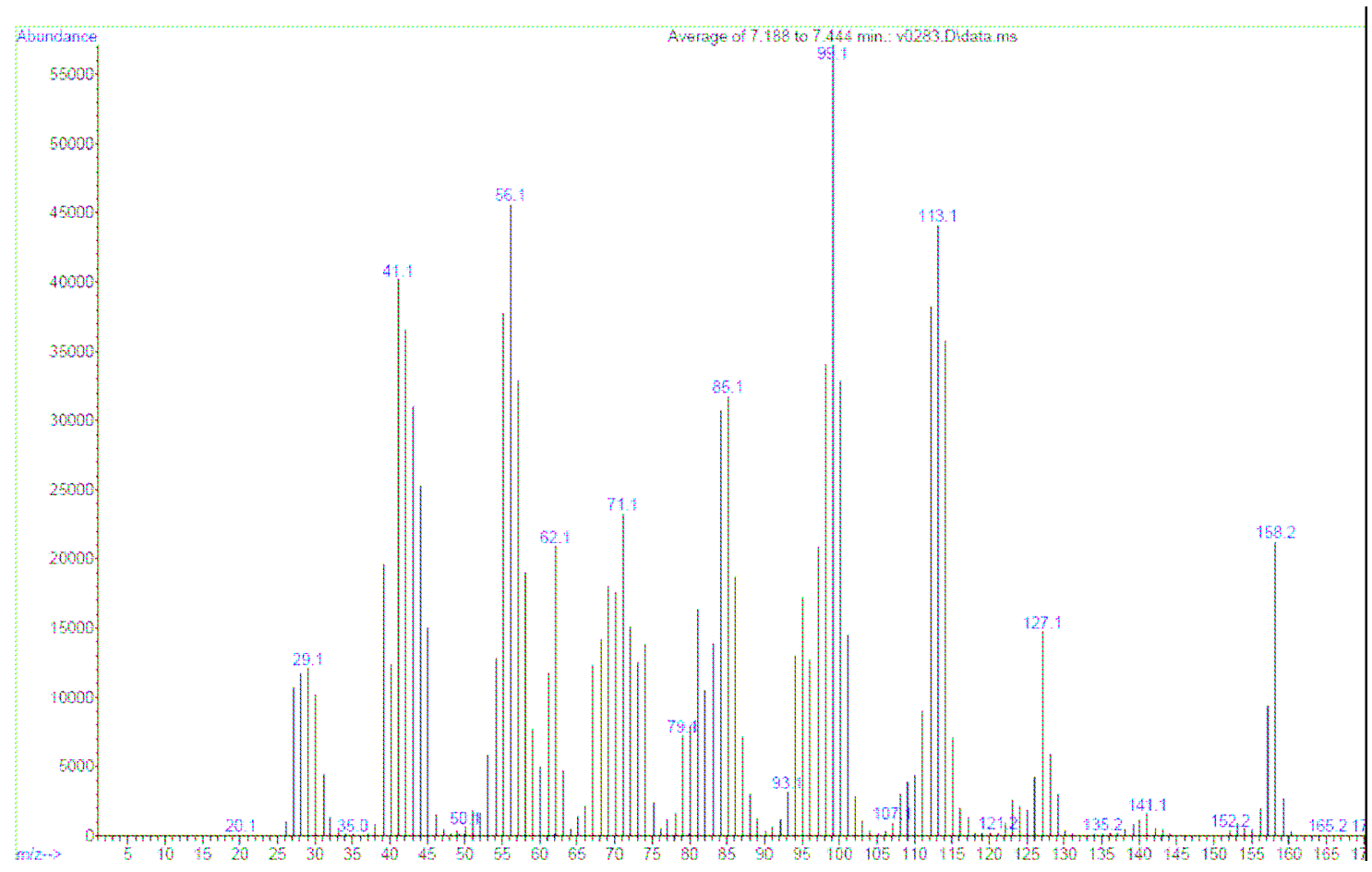




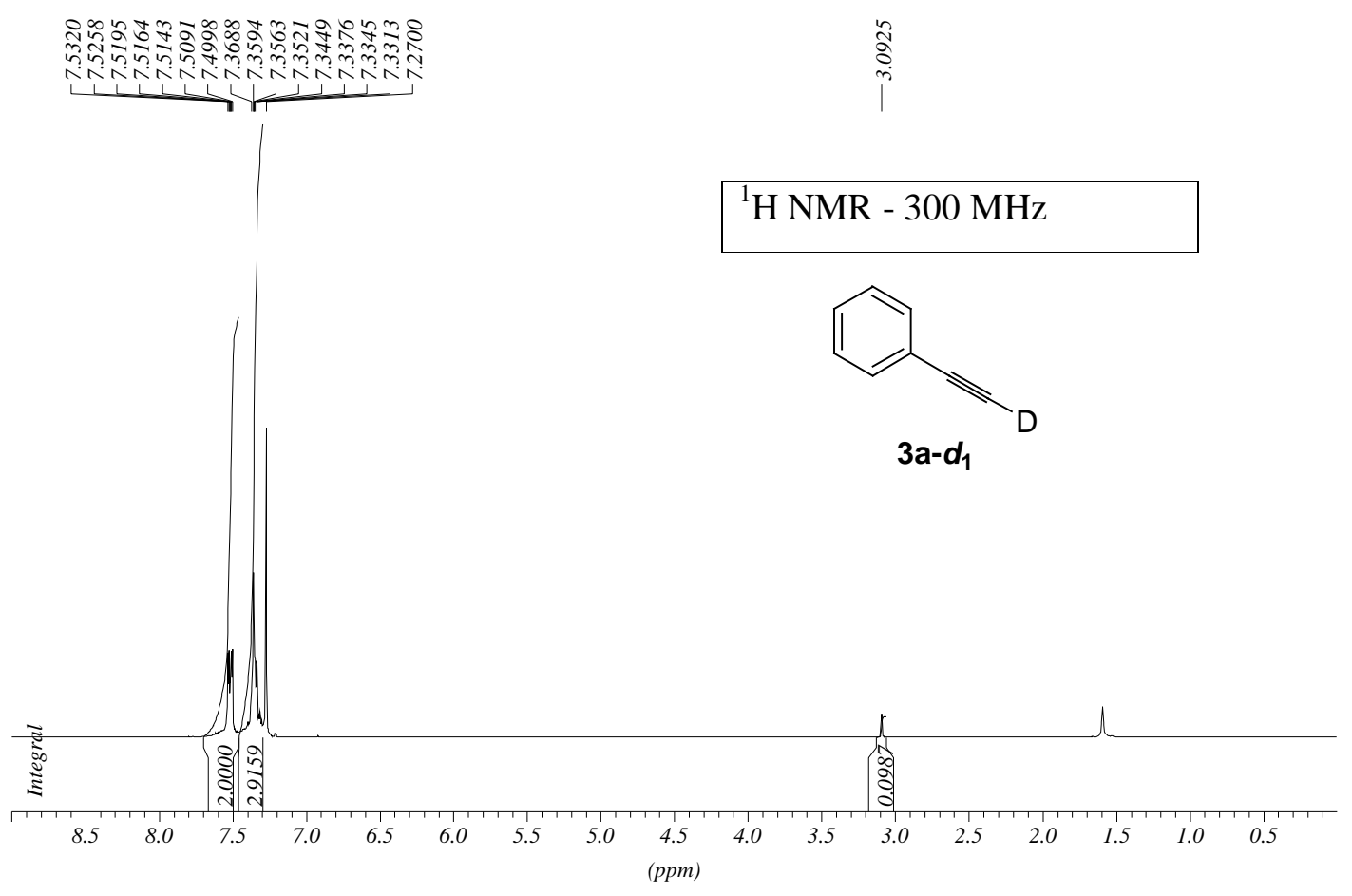



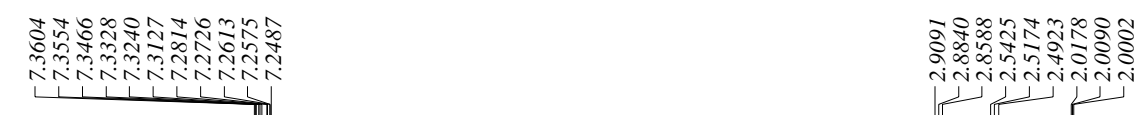

anon

INTNNTN

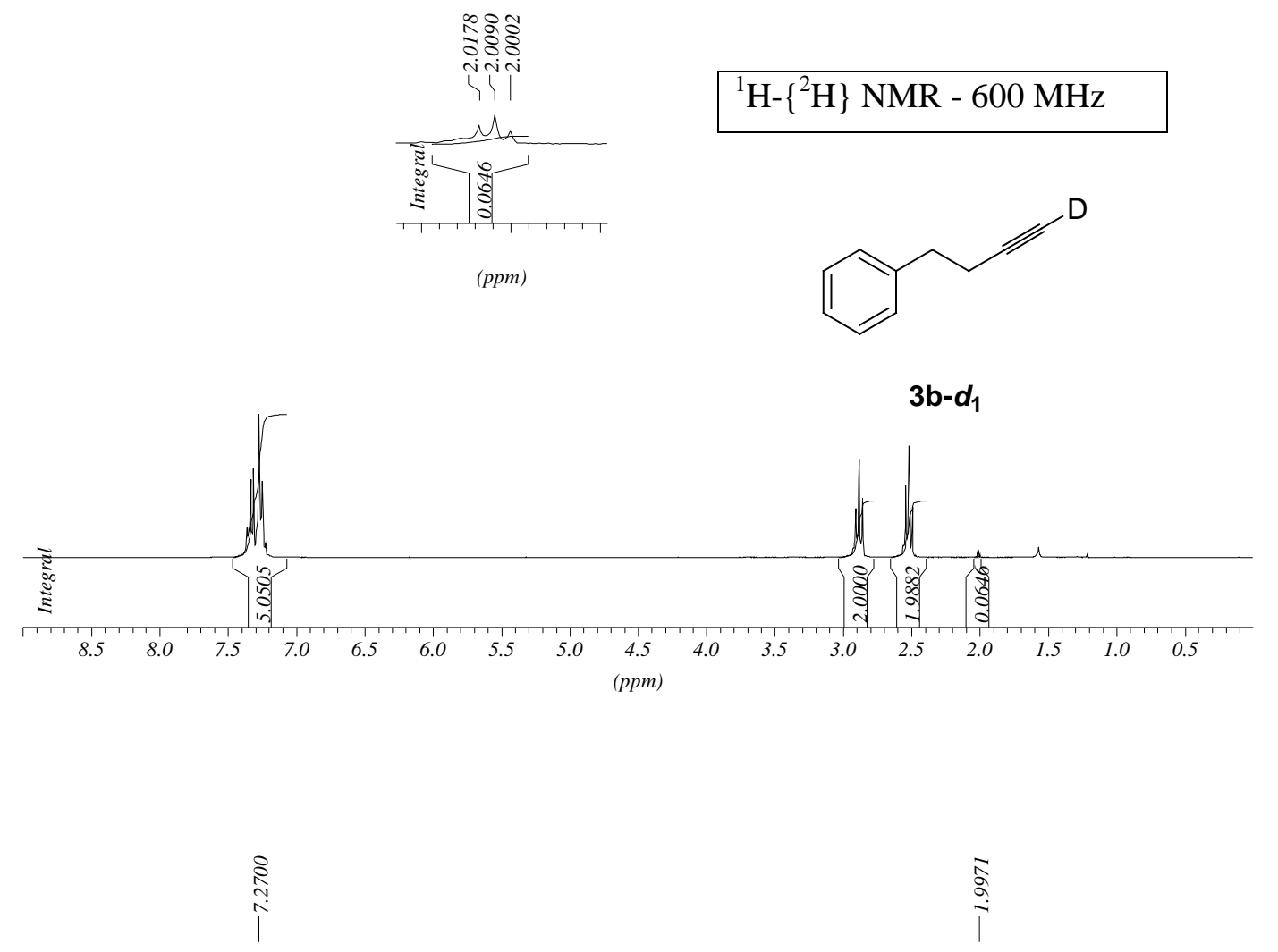

${ }^{2} \mathrm{H}$ NMR - $46 \mathrm{MHz}$

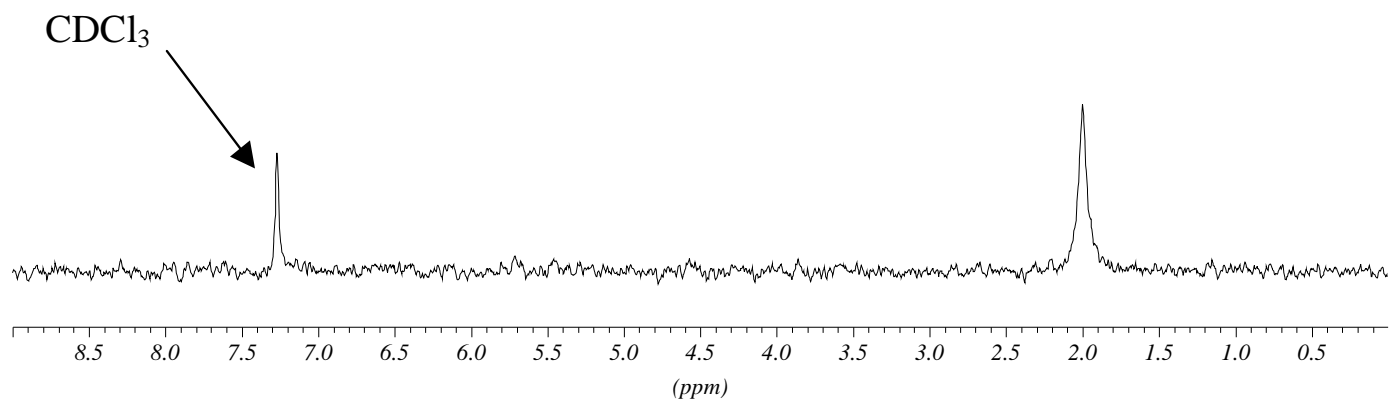




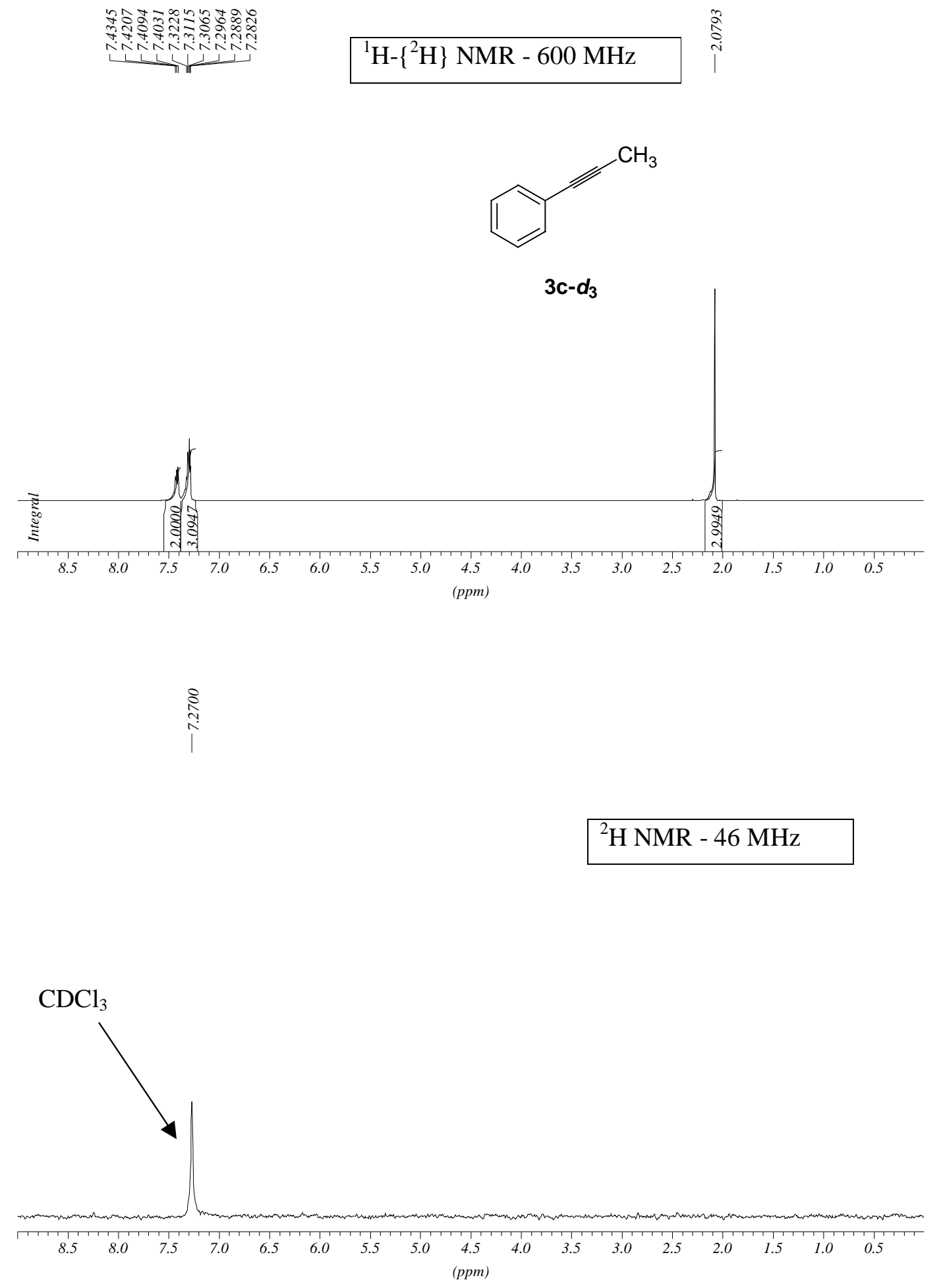


Reaction of $\mathbf{2 d}$ with TBD
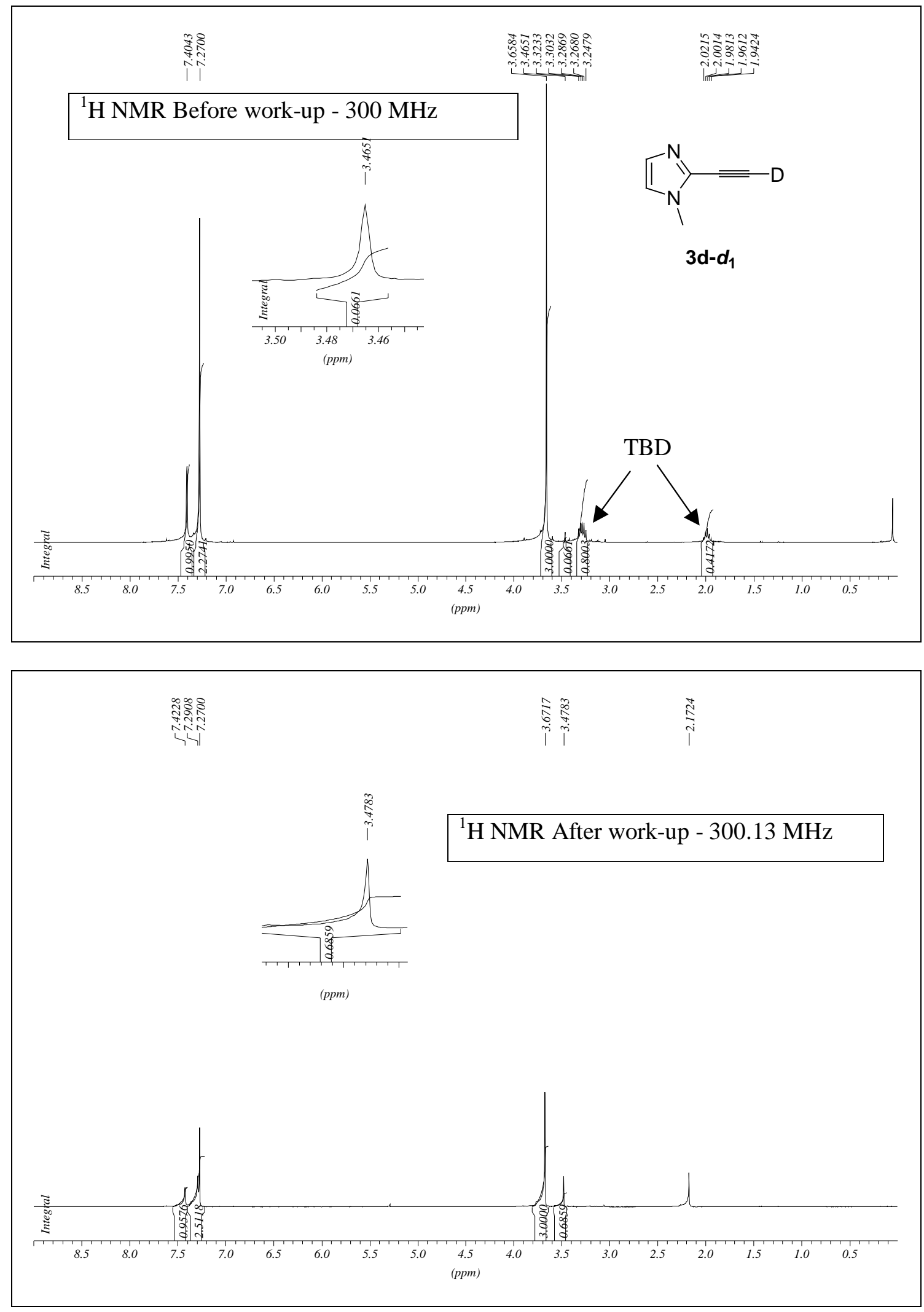
Reaction 2d with PSTBD

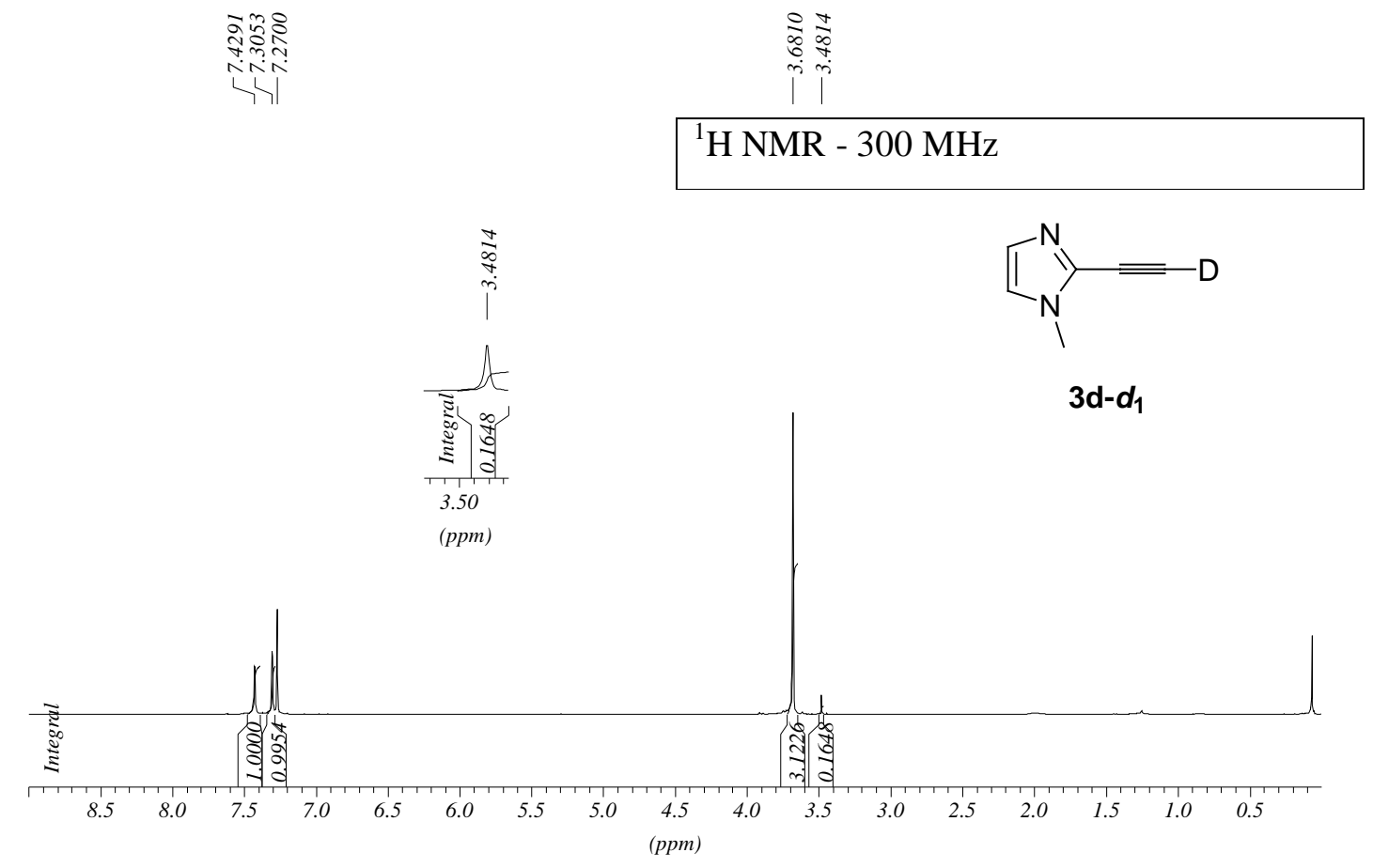



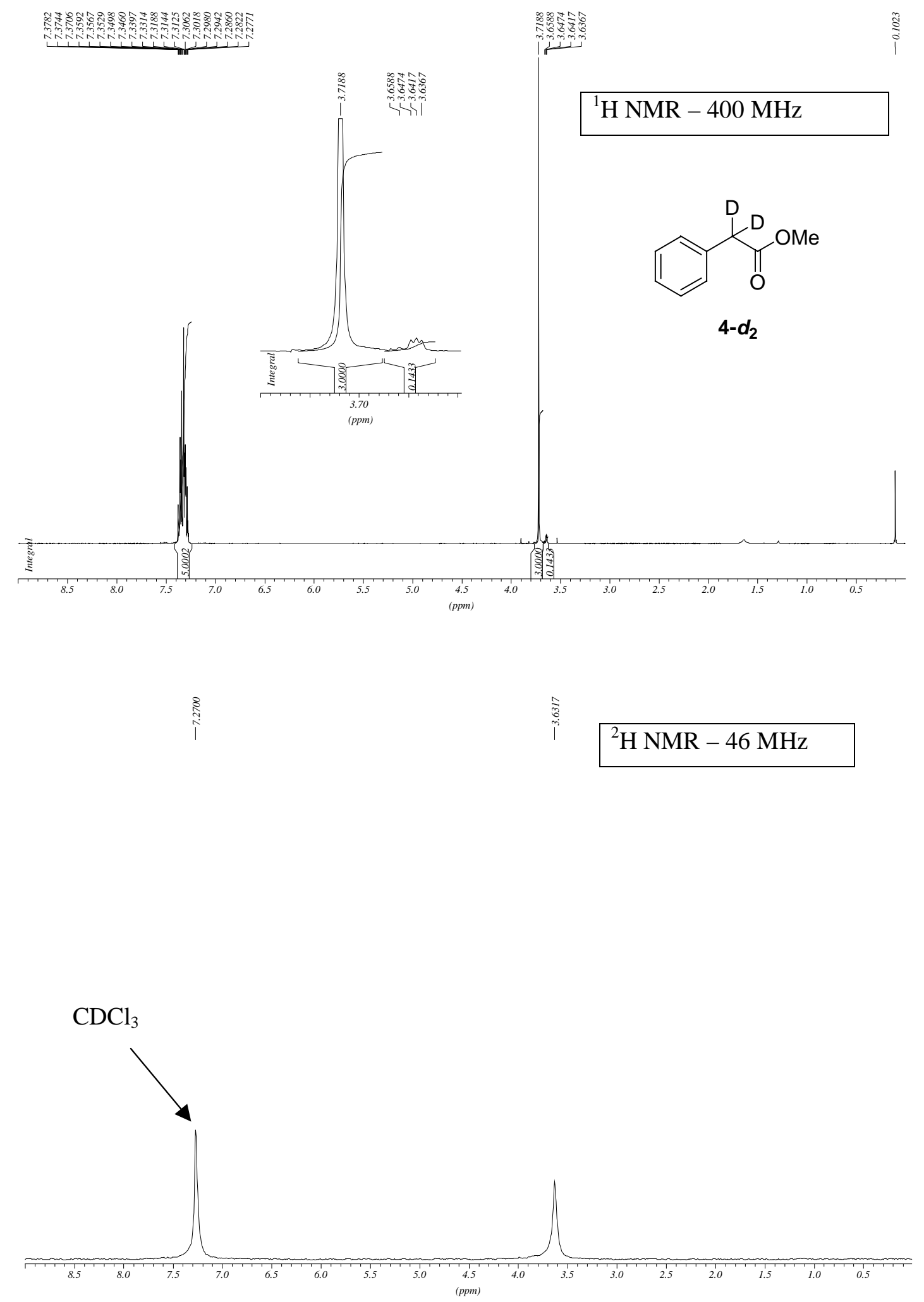

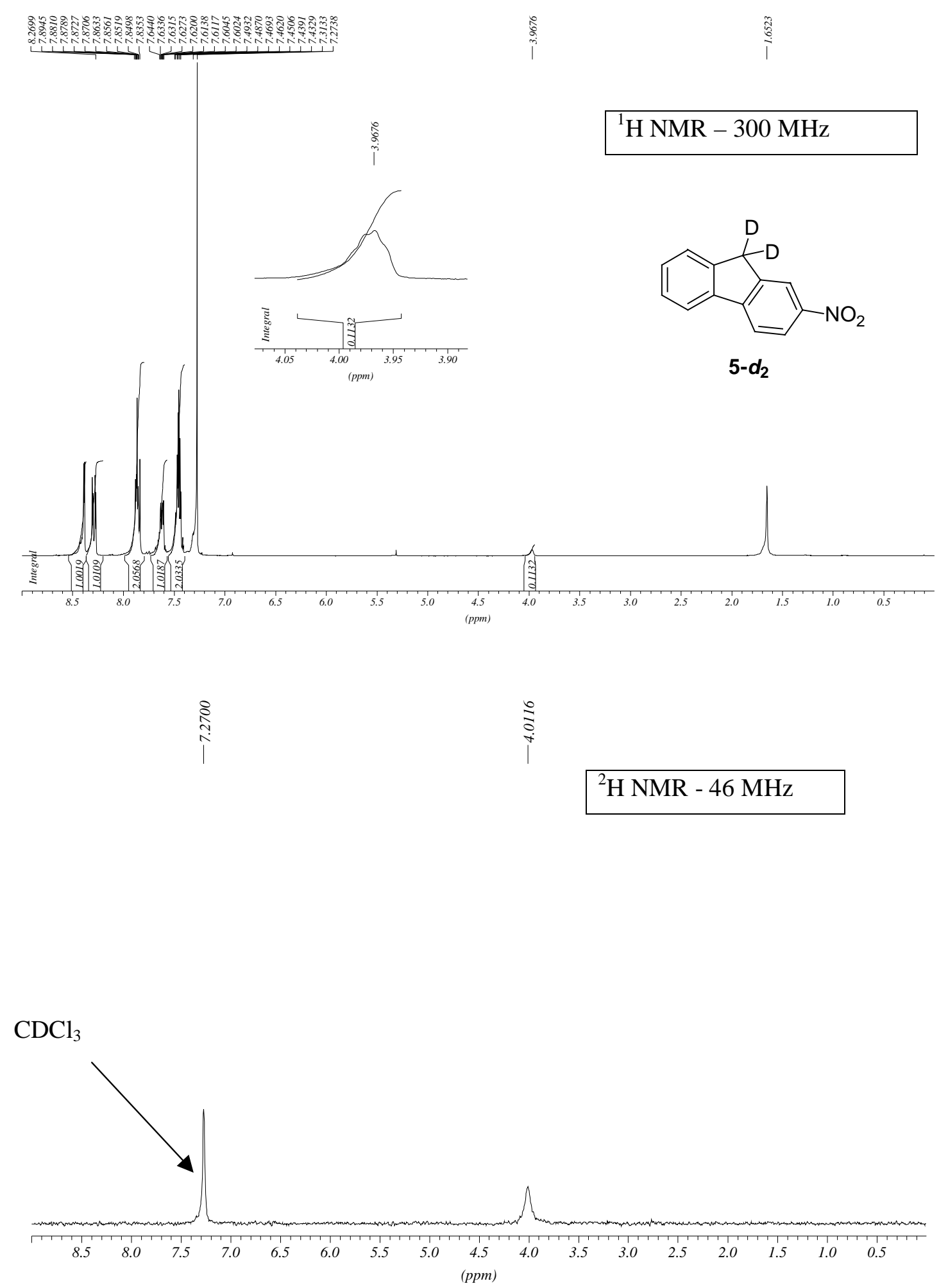


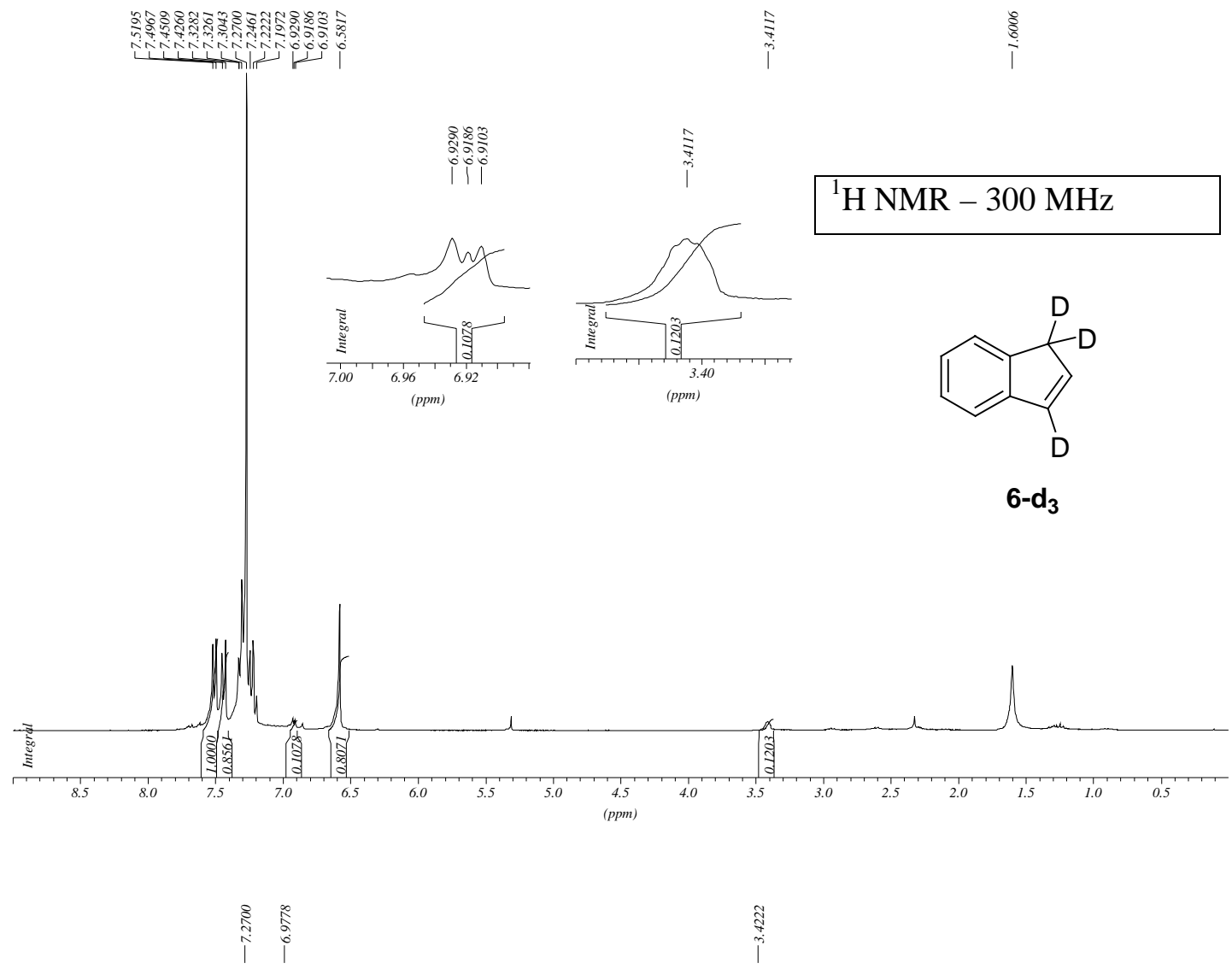

${ }^{2} \mathrm{H}$ NMR - $46 \mathrm{MHz}$

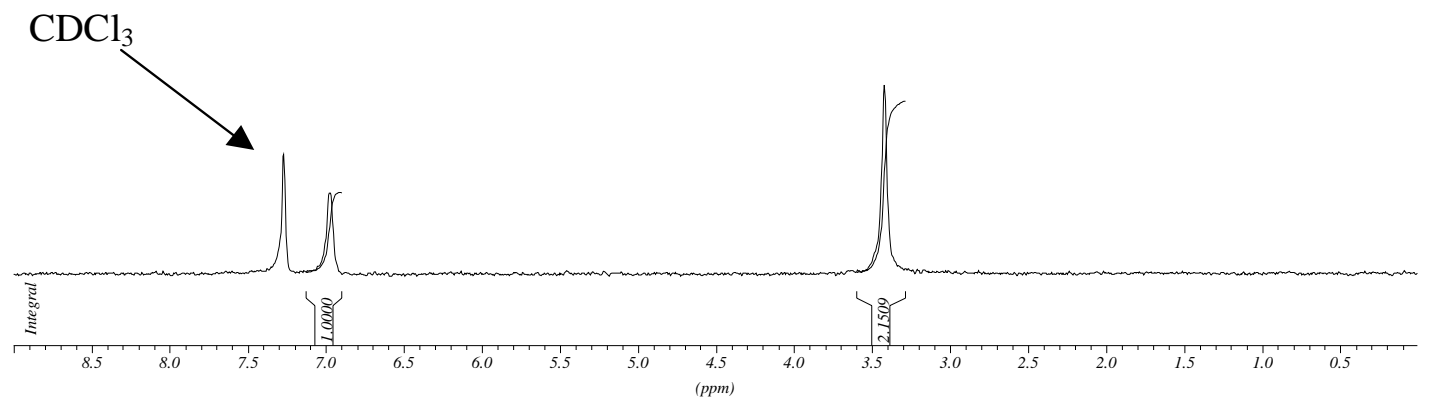




\section{Mechanistic study}
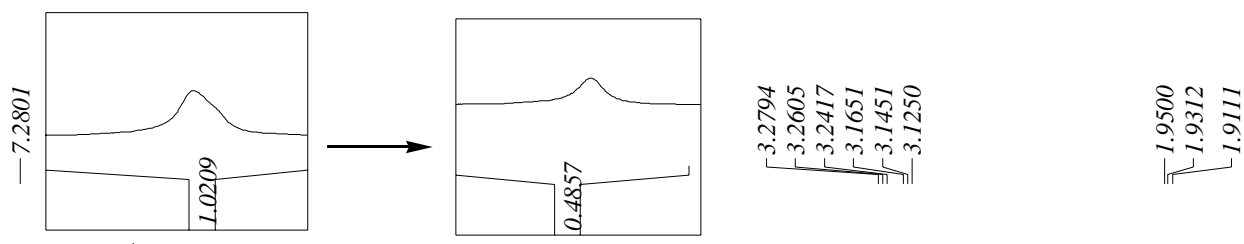

$\mathrm{C} / \mathrm{TBD}$ and $\mathrm{CDCl}_{3}$ (1 éq.) in $\mathrm{CD}_{2} \mathrm{Cl}_{2}$

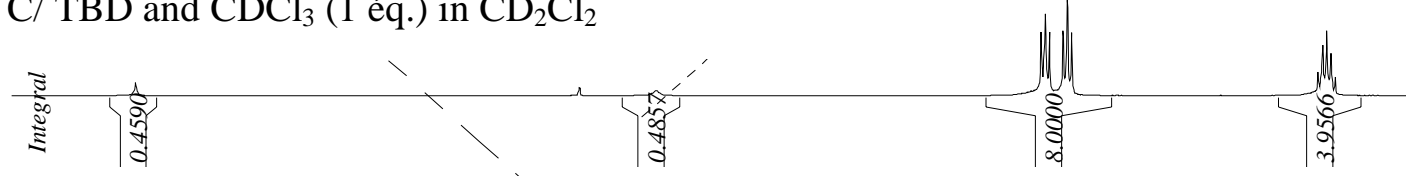

$\mathrm{B} / \mathrm{TBD}$ in $\mathrm{CD}_{2} \mathrm{Cl}_{2}$ :

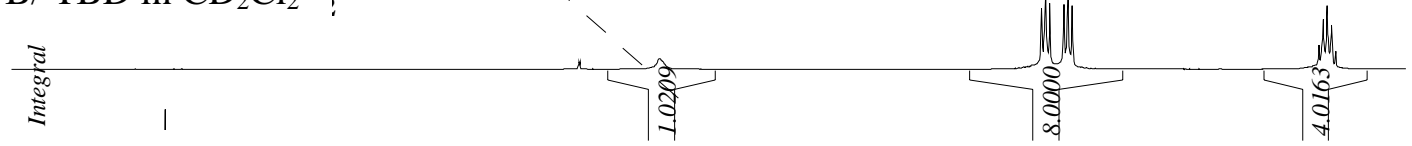

$\mathrm{A} / \mathrm{TBD}$ in $\mathrm{CDCl}_{3}$
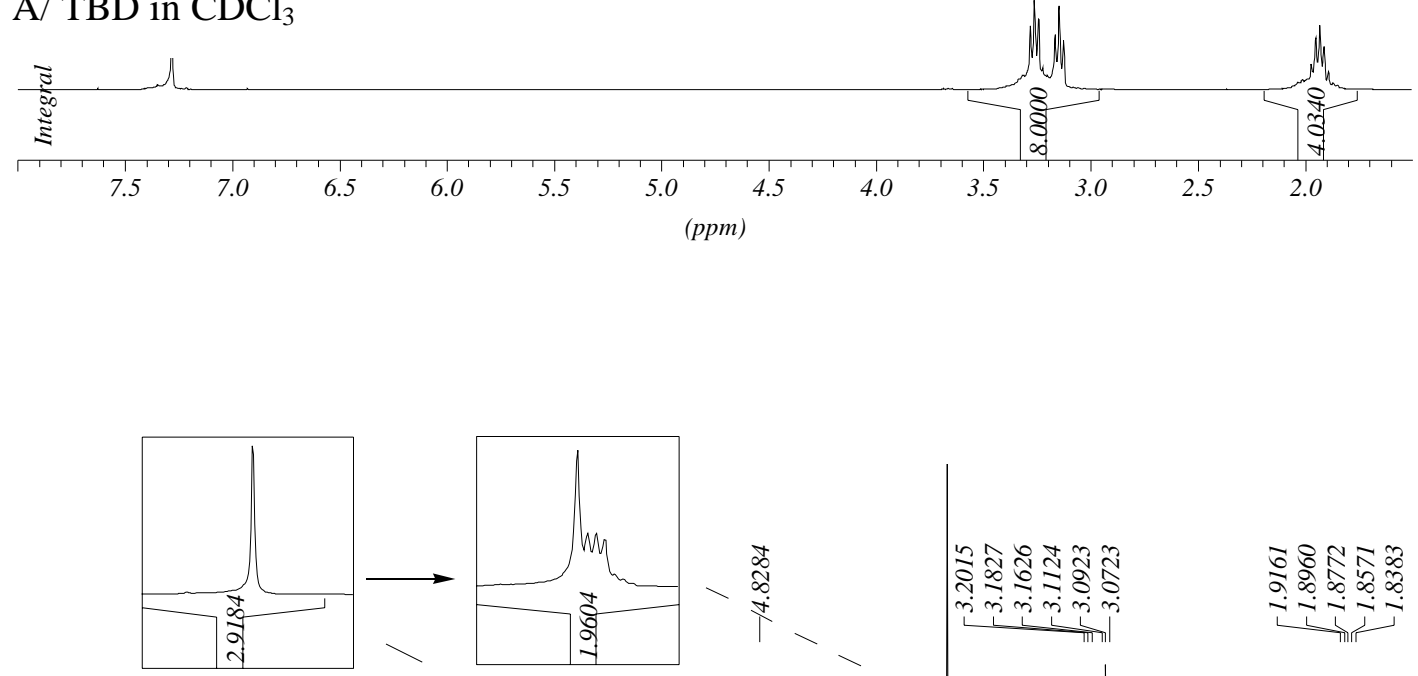

$\mathrm{C} / \mathrm{TBD}$ and 1a (1éq.) in $\mathrm{CD}_{2} \mathrm{Cl}_{2}$

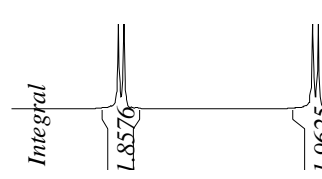

$\mathrm{B} /$ a1 in $\mathrm{CD}_{2} \mathrm{Cl}_{2}$

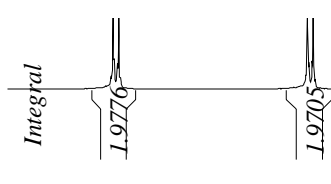

- A/ TBD- $d$ in $\mathrm{CD}_{2} \mathrm{Cl}_{2}$

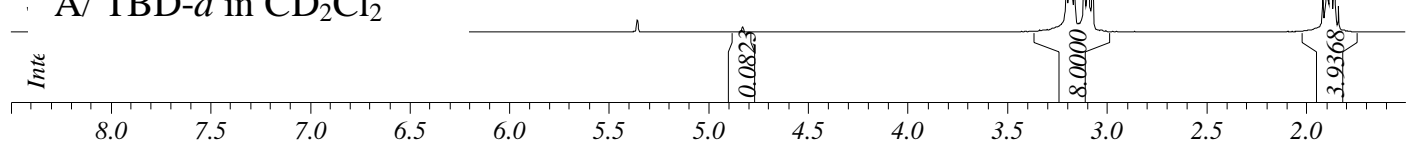




\section{References}

${ }^{1}$ Itsuno, I.; J. Chem. Soc. Perkin Trans. I 1985, 2039.

${ }^{2}$ Fujisaki, S.; Fujiwara, I.; Norisue, Y.; Kajigaeshi, S. Bull. Chem. Soc. Jpn. 1985, 58, 2429. 